\title{
Lorentzian Darboux images of curves on spacelike surfaces in Lorentz-Minkowski 3-space
}

\author{
Noriaki ITO and Shyuichi IZUMIYA
}

May 26, 2021

\begin{abstract}
For a regular curve on a spacelike surface in Lorentz-Minkowski 3-space, we have a moving frame along the curve which is called a Lorentzian Darboux frame. We introduce five special vector fields along the curve associated to the Lorentzian Darboux frame and investigate their singularities.
\end{abstract}

॥ा

\section{Introduction}

In this paper we consider a curve on a spacelike surface in the Lorentz-Minkowski 3-space and some special vector fields along the curve. The study of geometry of the Lorentz-Minkowski space is of interest in the special relativity theory. From the view point of mathematics, the interesting problem is how geometric properties of the Lorentz-Minkowski space is different from those of the Euclidean space. In the Euclidean 3-space, the notion of Darboux frames along curves on surfaces is well-known. In [6] spherical duals (cf. [2, 9]) of basis of the Darboux frame along a curve are introduced, which are called Darboux vector fields along the curve. There are three Darboux vector fields along the curve. Singularities and geometric properties of these three Darboux vectors were investigated in [6].

On the other hand, there also exists a Lorentzian version of Darboux frames along curves on spacelike surfaces [10]. We consider (pseudo-spherical) Legendrian duals (cf. [5, 7]) of basis of the Lorentzian Darboux frame along a curve, which are called Lorentzian Darboux vectors along the curve. Since there are three kinds of pseudo-spheres in Lorentz-Minkowski space, we have eight Lorentzian Darboux vectors along the curve. There are three Legendrian duals of the unit tangent vector along the curve, which were essentially investigated in [10]. Those vector fields are three of the Lorentzian Darboux vector fields along the curve. Therefore, we consider remaining five Lorentzian Darboux vectors along the curve here. We investigate the singularities of the pseudo-spherical image of Lorentzian Darboux vectors. As a consequence, we obtain five new Lorentzian invariants which characterize the singularities of these Lorentzian Darboux vectors. We also investigate the geometric meanings of these invariants.

2010 Mathematics Subject classification. Primary 53A04; Secondary 58Kxx

Key Words and Phrases. Lorentzian Darboux frame, Lorentzian Darboux vector field, pseudo-spherical image, singularities 


\section{Basic concepts}

d In this section we prepare some definitions and basic facts which we will use in this paper. For basic concepts and details of properties, see $\left[8,10\right.$. Let $\mathbb{R}^{3}$ be a three-dimensional vector space. For any $\boldsymbol{x}=\left(x_{0}, x_{1}, x_{2}\right), \boldsymbol{y}=\left(y_{0}, y_{1}, y_{2}\right) \in \mathbb{R}^{3}$, the pseudo-scalar product of $\boldsymbol{x}$ and $\boldsymbol{y}$ is defined by $\langle\boldsymbol{x}, \boldsymbol{y}\rangle=-x_{0} y_{0}+x_{1} y_{1}+x_{2} y_{2}$. We call $\left(\mathbb{R}^{3},\langle\rangle,\right)$ the Lorentz-Minkowski 3-space. We write $\mathbb{R}_{1}^{3}$ instead of $\left(\mathbb{R}^{3},\langle\rangle,\right)$. We say that a non-zero vector $\boldsymbol{x} \in \mathbb{R}_{1}^{3}$ is spacelike, lightlike or timelike if $\langle\boldsymbol{x}, \boldsymbol{x}\rangle>0,\langle\boldsymbol{x}, \boldsymbol{x}\rangle=0$ or $\langle\boldsymbol{x}, \boldsymbol{x}\rangle<0$, respectively. The norm of the vector $\boldsymbol{x} \in \mathbb{R}_{1}^{3}$ is defined by $\|\boldsymbol{x}\|=\sqrt{|\langle\boldsymbol{x}, \boldsymbol{x}\rangle|}$. For a non-zero vector $\boldsymbol{v} \in \mathbb{R}_{1}^{3}$ and a real number $c \in \mathbb{R}$, we define a plane with a pseudo-normal $\boldsymbol{v}$ by

$$
P(\boldsymbol{v}, c)=\left\{\boldsymbol{x} \in \mathbb{R}_{1}^{3} \mid\langle\boldsymbol{x}, \boldsymbol{v}\rangle=c\right\} .
$$

We call $P(\boldsymbol{v}, c)$ a spacelike plane, a timelike plane or a lightlike plane if $\boldsymbol{v}$ is timelike, spacelike or lightlike, respectively. We introduce three pseudo-spheres in $\mathbb{R}_{1}^{3}$ as follows: We define the hyperbolic plane by

$$
H^{2}(-1)=\left\{\boldsymbol{x} \in \mathbb{R}_{1}^{3} \mid\langle\boldsymbol{x}, \boldsymbol{x}\rangle=-1\right\},
$$

de Sitter 2-space by

$$
S_{1}^{2}=\left\{\boldsymbol{x} \in \mathbb{R}_{1}^{3} \mid\langle\boldsymbol{x}, \boldsymbol{x}\rangle=1\right\}
$$

and the (open) lightcone by

$$
L C^{*}=\left\{\boldsymbol{x} \in \mathbb{R}_{1}^{3} \backslash\{\boldsymbol{0}\} \mid\langle\boldsymbol{x}, \boldsymbol{x}\rangle=0\right\} .
$$

We also define the following curves on the pseudo-spheres with constant curvatures: A curve defined by the intersection of $H^{2}(-1)$ with a plane is called a hyperbolic line (respectively, a horocycle) if the plane is a timelike plane through the origin (respectively, a lightlike plane). We also say that a curve on the de Sitter 2-space $S_{1}^{2}$ is a geodesic pseudo-circle (respectively, a geodesic hyperbola) if it is defined by the intersection of $S_{1}^{2}$ with a spacelike (respectively, a timelike) plane through the origin. Moreover, a curve on $S_{1}^{2}$ is said to be a de Sitter horocycle if it is defined by the intersection of $S_{1}^{2}$ with a lightlike plane away from the origin. Here we define

$$
\boldsymbol{a} \wedge \boldsymbol{b}=\left|\begin{array}{ccc}
-\boldsymbol{e}_{0} & \boldsymbol{e}_{1} & \boldsymbol{e}_{2} \\
a_{0} & a_{1} & a_{2} \\
b_{0} & b_{1} & b_{2}
\end{array}\right|,
$$

where $\boldsymbol{a}=\left(a_{0}, a_{1}, a_{2}\right), \boldsymbol{b}=\left(b_{0}, b_{1}, b_{2}\right)$ and $\left\{\boldsymbol{e}_{0}, \boldsymbol{e}_{1}, \boldsymbol{e}_{2}\right\}$ is the canonical basis of $\mathbb{R}^{3}$.

We now prepare some basic facts of curves on a spacelike surface. We consider a spacelike embedding $\boldsymbol{X}: U \longrightarrow \mathbb{R}_{1}^{3}$ from an open subset $U \subset \mathbb{R}^{2}$. We write $M=\boldsymbol{X}(U)$ and identify $M$ and $U$ through the embedding $\boldsymbol{X}$. Here, we say that $\boldsymbol{X}$ is a spacelike embedding if the tangent space $T_{p} M$ consists of spacelike vectors at any $p=\boldsymbol{X}(u)$. Let $\bar{\gamma}: I \longrightarrow U$ be a regular curve and we have a curve $\boldsymbol{\gamma}: I \longrightarrow M \subset \mathbb{R}_{1}^{3}$ defined by $\boldsymbol{\gamma}(s)=\boldsymbol{X}(\overline{\boldsymbol{\gamma}}(s))$. We say that $\boldsymbol{\gamma}$ is a curve on the spacelike surface $M$. Since $\boldsymbol{\gamma}$ is a spacelike curve, we can reparametrize it by the arc-length $s$. So we have the spacelike unit tangent vector $\boldsymbol{t}(s)=\boldsymbol{\gamma}^{\prime}(s)$ of $\boldsymbol{\gamma}(s)$. Since $\boldsymbol{X}$ is a spacelike embedding, we have a timelike unit normal vector field $\boldsymbol{n}$ along $M=\boldsymbol{X}(U)$ defined by

$$
\boldsymbol{n}(p)=\frac{\boldsymbol{X}_{u_{1}}(u) \wedge \boldsymbol{X}_{u_{2}}(u)}{\left\|\boldsymbol{X}_{u_{1}}(u) \wedge \boldsymbol{X}_{u_{2}}(u)\right\|}
$$


for $p=\boldsymbol{X}(u)$. We say that $\boldsymbol{n}$ is future directed if $\left\langle\boldsymbol{n}, \boldsymbol{e}_{0}\right\rangle<0$. We choose the orientation of $M$ such that $\boldsymbol{n}$ is future directed. We define $\boldsymbol{n}_{\boldsymbol{\gamma}}(s)=\boldsymbol{n} \circ \boldsymbol{\gamma}(s)$, so that we have a timelike unit normal vector field $\boldsymbol{n}_{\boldsymbol{\gamma}}$ along $\boldsymbol{\gamma}$. Therefore we can construct a spacelike unit normal vetoer field $\boldsymbol{b}(s) \in N_{p}(M)$ defined by $\boldsymbol{b}(s)=\boldsymbol{t}(s) \wedge \boldsymbol{n}_{\gamma}(s)$. It follows that we have $\left\langle\boldsymbol{n}_{\gamma}, \boldsymbol{n}_{\gamma}\right\rangle=-1,\left\langle\boldsymbol{n}_{\gamma}, \boldsymbol{b}\right\rangle=$ $0,\langle\boldsymbol{b}, \boldsymbol{b}\rangle=1$. Then we have a pseudo-orthonormal frame $\left\{\boldsymbol{t}(s), \boldsymbol{n}_{\gamma}(s), \boldsymbol{b}(s)\right\}$ along $\boldsymbol{\gamma}$, which is called the Lorentzian Darboux frame along $\gamma$. By standard arguments, we have the following Frenet-Serret type formulae:

$$
\left\{\begin{array}{l}
\boldsymbol{t}^{\prime}(s)=\kappa_{n}(s) \boldsymbol{n}_{\gamma}(s)+\kappa_{g}(s) \boldsymbol{b}(s) \\
\boldsymbol{n}_{\gamma}^{\prime}(s)=\kappa_{n}(s) \boldsymbol{t}(s)+\tau_{g}(s) \boldsymbol{b}(s) \\
\boldsymbol{b}^{\prime}(s)=-\kappa_{g}(s) \boldsymbol{t}(s)+\tau_{g}(s) \boldsymbol{n}_{\gamma}(s)
\end{array}\right.
$$

where $\kappa_{n}(s)=-\left\langle\boldsymbol{t}^{\prime}(s), \boldsymbol{n}_{\gamma}(s)\right\rangle, \kappa_{g}(s)=\left\langle\boldsymbol{t}^{\prime}(s), \boldsymbol{b}(s)\right\rangle$ and $\tau_{g}(s)=-\left\langle\boldsymbol{b}^{\prime}(s), \boldsymbol{n}_{\gamma}(s)\right\rangle$. We have the geometric characterizations of $\gamma$ by the invariants $\kappa_{g}, \kappa_{n}$ and $\tau_{g}$. We say that $\gamma$ is a geodesic curve if the curvature vector $\boldsymbol{t}^{\prime}(s)$ has only a pseudo-normal component of the surface, an asymptotic curve if $\boldsymbol{t}^{\prime}(s)$ has only a tangential component of the surface and a principal curve if $\boldsymbol{n}_{\gamma}^{\prime}(s)$ is equal to the tangent direction of $\boldsymbol{\gamma}$, respectively. Then

$$
\gamma \text { is }\left\{\begin{array}{l}
\text { a geodesic curve if and only if } \kappa_{g} \equiv 0 \\
\text { an asymptotic curve if and only if } \kappa_{n} \equiv 0 \\
\text { a principal curve if and only if } \tau_{g} \equiv 0
\end{array}\right.
$$

Then we define the following five pseudo-spherical Lorentzian Darboux images along $\gamma$ :

$$
\begin{aligned}
& (A) \overline{\boldsymbol{D}}_{r}^{T}: I \longrightarrow H^{2}(-1) ; \overline{\boldsymbol{D}}_{r}^{T}(s)=\frac{\tau_{g}(s) \boldsymbol{t}(s)-\kappa_{g}(s) \boldsymbol{n}_{\boldsymbol{\gamma}}(s)}{\sqrt{\kappa_{g}(s)^{2}-\tau_{g}(s)^{2}}} \text { if } \kappa_{g}(s)^{2}>\tau_{g}(s)^{2}, \\
& (B) \overline{\boldsymbol{D}}_{r}^{S}: I \longrightarrow S_{1}^{2} ; \overline{\boldsymbol{D}}_{r}^{S}(s)=\frac{\tau_{g}(s) \boldsymbol{t}(s)-\kappa_{g}(s) \boldsymbol{n}_{\boldsymbol{\gamma}}(s)}{\sqrt{\tau_{g}(s)^{2}-\kappa_{g}(s)^{2}}} \text { if } \tau_{g}(s)^{2}>\kappa_{g}(s)^{2}, \\
& (C) \overline{\boldsymbol{D}}_{r}^{L}: I \longrightarrow L C^{*} ; \overline{\boldsymbol{D}}_{r}^{L}(s)=\frac{\tau_{g}(s) \boldsymbol{t}(s)-\kappa_{g}(s) \boldsymbol{n}_{\boldsymbol{\gamma}}(s)}{\sqrt{\kappa_{g}(s)^{2}-\tau_{g}(s)^{2}}}+\boldsymbol{b}(s) \text { if } \kappa_{g}(s)^{2}>\tau_{g}(s)^{2}, \\
& \text { (D) } \overline{\boldsymbol{D}}_{o}^{S}: I \longrightarrow S_{1}^{2} ; \overline{\boldsymbol{D}}_{o}^{S}(s)=\frac{\tau_{g}(s) \boldsymbol{t}(s)-\kappa_{n}(s) \boldsymbol{b}(s)}{\sqrt{\kappa_{n}(s)^{2}+\tau_{g}(s)^{2}}} \text { if }\left(\kappa_{n}(s), \tau_{g}(s)\right) \neq(0,0), \\
& \text { (E) } \overline{\boldsymbol{D}}_{o}^{L}: I \longrightarrow L C^{*} ; \overline{\boldsymbol{D}}_{o}^{L}(s)=\frac{\tau_{g}(s) \boldsymbol{t}(s)-\kappa_{n}(s) \boldsymbol{b}(s)}{\sqrt{\kappa_{n}(s)^{2}+\tau_{g}(s)^{2}}}+\boldsymbol{n}_{\boldsymbol{\gamma}}(s) \text { if }\left(\kappa_{n}(s), \tau_{g}(s)\right) \neq(0,0) .
\end{aligned}
$$

We call $(A)$ the pseudo-spherical rectifying timelike Darboux image, $(B)$ the pseudo-spherical rectifying spacelike Darboux image, $(C)$ the pseudo-spherical rectifying lightlike Darboux image, $(D)$ the pseudo-spherical osculating spacelike Darboux image and $(E)$ the pseudo-spherical osculating lightlike Darboux image along $\boldsymbol{\gamma}$, respectively. We remark that we cannot define a pseudo-spherical osculating timelike Darboux image. 
Remark 2.1 We can define extra three pseudo-spherical Lorentzian Darboux images along $\gamma$ :

$$
\begin{aligned}
& (F) \overline{\boldsymbol{D}}_{n}^{T}: I \longrightarrow H^{2}(-1) ; \overline{\boldsymbol{D}}_{n}^{T}(s)=\frac{\kappa_{g} \boldsymbol{n}_{\boldsymbol{\gamma}}(s)+\kappa_{n}(s) \boldsymbol{b}(s)}{\sqrt{\kappa_{g}(s)^{2}-\kappa_{n}(s)^{2}}} \text { if } \kappa_{g}(s)^{2}>\kappa_{n}(s)^{2}, \\
& (G) \overline{\boldsymbol{D}}_{n}^{S}: I \longrightarrow S_{1}^{2} ; \overline{\boldsymbol{D}}_{n}^{S}(s)=\frac{\kappa_{g} \boldsymbol{n}_{\boldsymbol{\gamma}}(s)+\kappa_{n}(s) \boldsymbol{b}(s)}{\sqrt{\kappa_{n}(s)^{2}-\kappa_{g}(s)^{2}}} \text { if } \kappa_{n}(s)^{2}>\kappa_{g}(s)^{2}, \\
& (H) \overline{\boldsymbol{D}}_{n}^{L}: I \longrightarrow L C^{*} ; \overline{\boldsymbol{D}}_{n}^{L}(s)=\frac{\kappa_{g} \boldsymbol{n}_{\boldsymbol{\gamma}}(s)+\kappa_{n}(s) \boldsymbol{b}(s)}{\sqrt{\kappa_{g}(s)^{2}-\kappa_{n}(s)^{2}}}+\boldsymbol{t}(s) \text { if } \kappa_{g}(s)^{2}>\kappa_{n}(s)^{2},
\end{aligned}
$$

Singularities and geometric meanings of $(F)$ and $(G)$ were investigated [10]. Moreover, we can easily obtain the similar results for $(\mathrm{H})$, so that $(\mathrm{H})$ was also essentially investigated in [10]. Therefore we omit the investigations on those three cases here.

\section{Singularities of pseudo-spherical Lorentzian Darboux images}

In this section we present a classification result of the singularities of pseudo-spherical Lorentzian Darboux images.

We now introduce five invariants of $(M, \gamma)$ as follows:
(A) $\delta_{r}^{T}(s)=\kappa_{n}(s)-\frac{\kappa_{g}(s) \tau_{g}(s)^{\prime}-\kappa_{g}(s)^{\prime} \tau_{g}(s)}{\kappa_{g}(s)^{2}-\tau_{g}(s)^{2}}$ if $\kappa_{g}(s)^{2}>\tau_{g}(s)^{2}$,
$(B) \delta_{r}^{S}(s)=\kappa_{n}(s)+\frac{\kappa_{g}(s) \tau_{g}(s)^{\prime}-\kappa_{g}(s)^{\prime} \tau_{g}(s)}{\tau_{g}(s)^{2}-\kappa_{g}(s)^{2}}$ if $\tau_{g}(s)^{2}>\kappa_{g}(s)^{2}$,
$(C) \delta_{r}^{L}(s)=\kappa_{n}(s)-\frac{\kappa_{g}(s) \tau_{g}(s)^{\prime}-\kappa_{g}(s)^{\prime} \tau_{g}(s)}{\kappa_{g}(s)^{2}-\tau_{g}(s)^{2}}+\sqrt{\kappa_{g}(s)^{2}-\tau_{g}(s)^{2}} \kappa_{g}(s)^{2}>\tau_{g}(s)^{2}$,
(D) $\delta_{o}^{S}(s)=\kappa_{g}(s)+\frac{\kappa_{n}(s) \tau_{g}(s)^{\prime}-\kappa_{n}(s)^{\prime} \tau_{g}(s)}{\kappa_{n}(s)^{2}+\tau_{g}(s)^{2}}$ if $\left(\kappa_{n}(s), \tau_{g}(s)\right) \neq(0,0)$,
$(E) \delta_{o}^{L}(s)=\kappa_{g}(s)+\frac{\kappa_{n}(s) \tau_{g}(s)^{\prime}-\kappa_{n}(s)^{\prime} \tau_{g}(s)}{\kappa_{n}(s)^{2}+\tau_{g}(s)^{2}}+\sqrt{\kappa_{n}(s)^{2}+\tau_{g}(s)^{2}}$ if $\left(\kappa_{n}(s), \tau_{g}(s)\right) \neq(0,0)$.

We can classify the singular points of pseudo-spherical Lorentzian Darboux images by using the above invariants.

Theorem 3.1 Let $\gamma: I \longrightarrow M$ be a unit speed curve on a spacelike surface $M \subset \mathbb{R}_{1}^{3}$ such that $\left\|\boldsymbol{t}^{\prime}(s)\right\| \neq 0$ and $\left\|\boldsymbol{b}^{\prime}(s)\right\| \neq 0$.

(A) Suppose that $\kappa_{g}^{2}\left(s_{0}\right)>\tau_{g}^{2}\left(s_{0}\right)$. Then we have the following assertions:

(1) $\overline{\boldsymbol{D}}_{r}^{T}$ is non-singular at $s_{0}$ if and only if $\delta_{r}^{T}\left(s_{0}\right) \neq 0$.

(2) The image of $\overline{\boldsymbol{D}}_{r}^{T}$ is locally diffeomorphic to the ordinary cusp $C$ at $s_{0}$ if and only if $\delta_{r}^{T}\left(s_{0}\right)=0$ and $\left(\delta_{r}^{T}\right)^{\prime}\left(s_{0}\right) \neq 0$

(B) Suppose that $\tau_{g}^{2}\left(s_{0}\right)>\kappa_{g}^{2}\left(s_{0}\right)$. Then we have the following assertions:

(1) $\overline{\boldsymbol{D}}_{r}^{S}$ is non-singular at $s_{0}$ if and only if $\delta_{r}^{S}\left(s_{0}\right) \neq 0$. 
(2) The image of $\overline{\boldsymbol{D}}_{r}^{S}$ is locally diffeomorphic to the ordinary cusp $C$ at $s_{0}$ if and only if $\delta_{r}^{S}\left(s_{0}\right)=0$ and $\left(\delta_{r}^{S}\right)^{\prime}\left(s_{0}\right) \neq 0$.

(C) Suppose that $\kappa_{g}^{2}\left(s_{0}\right)>\tau_{g}^{2}\left(s_{0}\right)$. Then we have the following assertions:

(1) $\overline{\boldsymbol{D}}_{r}^{L}$ is non-singular at $s_{0}$ if and only if $\delta_{r}^{L}\left(s_{0}\right) \neq 0$.

(2) The image of $\overline{\boldsymbol{D}}_{r}^{L}$ is locally diffeomorphic to the ordinary cusp $C$ at $s_{0}$ if and only if $\delta_{r}^{L}\left(s_{0}\right)=0$ and $\left(\delta_{r}^{L}\right)^{\prime}\left(s_{0}\right) \neq 0$.

(D) Suppose that $\left(\kappa_{n}(s), \tau_{g}(s)\right) \neq(0,0)$. Then we have the following assertions:

(1) $\overline{\boldsymbol{D}}_{o}^{S}$ is non-singular at $s_{0}$ if and only if $\delta_{o}^{S}\left(s_{0}\right) \neq 0$.

(2) The image of $\overline{\boldsymbol{D}}_{o}^{S}$ is locally diffeomorphic to the ordinary cusp $C$ at $s_{0}$ if and only if $\delta_{o}^{S}\left(s_{0}\right)=0$ and $\left(\delta_{o}^{S}\right)^{\prime}\left(s_{0}\right) \neq 0$.

(E) Suppose that $\left(\kappa_{n}(s), \tau_{g}(s)\right) \neq(0,0)$. Then we have the following assertions:

(1) $\overline{\boldsymbol{D}}_{o}^{L}$ is non-singular at $s_{0}$ if and only if $\delta_{o}^{L}\left(s_{0}\right) \neq 0$.

(2) The image of $\overline{\boldsymbol{D}}_{o}^{L}$ is locally diffeomorphic to the ordinary cusp $C$ at $s_{0}$ if and only if $\delta_{o}^{L}\left(s_{0}\right)=0$ and $\left(\delta_{o}^{L}\right)^{\prime}\left(s_{0}\right) \neq 0$.

Here, $C=\left\{\left(x_{1}, x_{2}\right) \mid x_{1}^{2}=x_{2}^{3}\right\}$ is the ordinary cusp (or, the semi-cubic parabola).

\section{Legendrian dualities}

We now review some properties of contact manifolds and Legendrian submanifolds. Let $N$ be a $(2 n+1)$-dimensional smooth manifold and $K$ be a tangent hyperplane field on $N$. Locally such a field is defined as the field of zeros of a 1 -form $\alpha$. The tangent hyperplane field $K$ is nondegenerate if $\alpha \wedge(d \alpha)^{n} \neq 0$ at any point of $N$. We say that $(N, K)$ is a contact manifold if $K$ is a non-degenerate hyperplane field. In this case $K$ is called a contact structure and $\alpha$ is a contact form. Let $\phi: N \longrightarrow N^{\prime}$ be a diffeomorphism between contact manifolds $(N, K)$ and $\left(N^{\prime}, K^{\prime}\right)$. We say that $\phi$ is a contact diffeomorphism if $d \phi(K)=K^{\prime}$. Two contact manifolds $(N, K)$ and $\left(N^{\prime}, K^{\prime}\right)$ are contact diffeomorphic if there exists a contact diffeomorphism $\phi: N \longrightarrow N^{\prime}$. A submanifold $i: L \subset N$ of a contact manifold $(N, K)$ is said to be Legendrian if $\operatorname{dim} L=n$ and $d i_{x}\left(T_{x} L\right) \subset K_{i(x)}$ at any $x \in L$. We say that a smooth fiber bundle $\pi: E \longrightarrow M$ is called a Legendrian fibration if its total space $E$ is furnished with a contact structure and its fibers are Legendrian submanifolds. Let $\pi: E \longrightarrow M$ be a Legendrian fibration. For a Legendrian submanifold $i: L \subset E, \pi \circ i: L \longrightarrow M$ is called a Legendrian map. The image of the Legendrian map $\pi \circ i$ is called a wavefront set of $i$ which is denoted by $W(L)$. For any $z \in E$, it is known that there is a local coordinate system $(x, p, y)=\left(x_{1}, \ldots, x_{m}, p_{1}, \ldots, p_{m}, y\right)$ around $z$ such that $\pi(x, p, y)=(x, y)$ and the contact structure is given by the 1 -form $\alpha=d y-\sum_{i=1}^{m} p_{i} d x_{i}$ (cf. [1], 20.3).

In [7] we have shown the basic duality theorem which is the fundamental tool for the study of spacelike hypersurfaces in Lorentz-Minkowski pseudo-spheres. We consider the following four double fibrations:

(1) (a) $H^{2}(-1) \times S_{1}^{2} \supset \Delta_{1}=\{(\boldsymbol{v}, \boldsymbol{w}) \mid\langle\boldsymbol{v}, \boldsymbol{w}\rangle=0\}$

(b) $\pi_{11}: \Delta_{1} \longrightarrow H^{2}(-1), \pi_{12}: \Delta_{1} \longrightarrow S_{1}^{2}$,

(c) $\theta_{11}=\langle d \boldsymbol{v}, \boldsymbol{w}\rangle\left|\Delta_{1}, \theta_{12}=\langle\boldsymbol{v}, d \boldsymbol{w}\rangle\right| \Delta_{1}$.

(2) (a) $H^{2}(-1) \times L C^{*} \supset \Delta_{2}=\{(\boldsymbol{v}, \boldsymbol{w}) \mid\langle\boldsymbol{v}, \boldsymbol{w}\rangle=-1\}$, 
(b) $\pi_{21}: \Delta_{2} \longrightarrow H^{2}(-1), \pi_{22}: \Delta_{2} \longrightarrow L C^{*}$,

(c) $\theta_{21}=\langle d \boldsymbol{v}, \boldsymbol{w}\rangle\left|\Delta_{2}, \theta_{22}=\langle\boldsymbol{v}, d \boldsymbol{w}\rangle\right| \Delta_{2}$.

(3) (a) $L C^{*} \times S_{1}^{2} \supset \Delta_{3}=\{(\boldsymbol{v}, \boldsymbol{w}) \mid\langle\boldsymbol{v}, \boldsymbol{w}\rangle=1\}$,

(b) $\pi_{31}: \Delta_{3} \longrightarrow L C^{*}, \pi_{32}: \Delta_{3} \longrightarrow S_{1}^{2}$,

(c) $\theta_{31}=\langle d \boldsymbol{v}, \boldsymbol{w}\rangle\left|\Delta_{3}, \theta_{32}=\langle\boldsymbol{v}, d \boldsymbol{w}\rangle\right| \Delta_{3}$.

(4) (a) $L C^{*} \times L C^{*} \supset \Delta_{4}=\{(\boldsymbol{v}, \boldsymbol{w}) \mid\langle\boldsymbol{v}, \boldsymbol{w}\rangle=-2\}$,

(b) $\pi_{41}: \Delta_{4} \longrightarrow L C^{*}, \pi_{42}: \Delta_{4} \longrightarrow L C^{*}$,

(c) $\theta_{41}=\langle d \boldsymbol{v}, \boldsymbol{w}\rangle\left|\Delta_{4}, \theta_{42}=\langle\boldsymbol{v}, d \boldsymbol{w}\rangle\right| \Delta_{4}$.

Here, $\pi_{i 1}(\boldsymbol{v}, \boldsymbol{w})=\boldsymbol{v}, \pi_{i 2}(\boldsymbol{v}, \boldsymbol{w})=\boldsymbol{w},\langle d \boldsymbol{v}, \boldsymbol{w}\rangle=-w_{0} d v_{0}+\sum_{i=1}^{2} w_{i} d v_{i}$ and $\langle\boldsymbol{v}, d \boldsymbol{w}\rangle=$ $-v_{0} d w_{0}+\sum_{i=1}^{2} v_{i} d w_{i}$.

We remark that $\theta_{i 1}^{-1}(0)$ and $\theta_{i 2}^{-1}(0)$ define the same tangent hyperplane field over $\Delta_{i}$ which is denoted by $K_{i}$. The basic duality theorem is the following theorem [7]:

Theorem 4.1 With the same notations as the previous paragraph, each $\left(\Delta_{i}, K_{i}\right)(i=1,2,3,4)$ is a contact manifold and both of $\pi_{i j}(j=1,2)$ are Legendrian fibrations. Moreover those contact manifolds are contact diffeomorphic each other.

Moreover, we have the following extra double fibration:
(a) $S_{1}^{2} \times S_{1}^{2} \supset \Delta_{5}=\{(\boldsymbol{v}, \boldsymbol{w}) \mid\langle\boldsymbol{v}, \boldsymbol{w}\rangle=0\}$,
(b) $\pi_{51}: \Delta_{5} \longrightarrow S_{1}^{2}, \pi_{52}: \Delta_{1} \longrightarrow S_{1}^{2}$,
(c) $\theta_{51}=\langle d \boldsymbol{v}, \boldsymbol{w}\rangle\left|\Delta_{5}, \theta_{52}=\langle\boldsymbol{v}, d \boldsymbol{w}\rangle\right| \Delta_{5}$.

It is shown in [5] that $\left(\Delta_{5}, K_{5}\right)$ is a contact manifold such that $\pi_{5 j}: \Delta_{5} \longrightarrow S_{1}^{2}, j=1,2$, are Legendrian fibrations which is not contact diffeomorphic to any other $\left(\Delta_{i}, K_{i}\right), i=1,2,3,4$. Given a Legendrian submanifold $i: L \rightarrow \Delta_{i}, i=1,2,3,4,5$, We say that $\pi_{i 1}(i(L))$ is the $\Delta_{i}$-dual of $\pi_{i 2}(i(L))$ and vice-versa.

Then we have the following duality theorem.

Theorem 4.2 Let $\gamma: I \longrightarrow M$ be a unit speed curve on a spacelike surface $M \subset \mathbb{R}_{1}^{3}$ such that $\left\|\boldsymbol{t}^{\prime}(s)\right\| \neq 0$ and $\left\|\boldsymbol{b}^{\prime}(s)\right\| \neq 0$.

(1) If $\left(\kappa_{n}(s), \tau_{g}(s)\right) \neq(0,0)$, then $\boldsymbol{n}_{\boldsymbol{\gamma}}$ is a $\Delta_{1}$-dual of $\overline{\boldsymbol{D}}_{o}^{S}$.

(2) If $\left(\kappa_{n}(s), \tau_{g}(s)\right) \neq(0,0)$, then $\boldsymbol{n}_{\boldsymbol{\gamma}}$ is a $\Delta_{2}$-dual of $\overline{\boldsymbol{D}}_{o}^{L}$.

(3) If $\kappa_{g}(s)^{2}>\tau_{g}(s)^{2}$, then $\boldsymbol{b}$ is a $\Delta_{1}$-dual of $\overline{\boldsymbol{D}}_{r}^{T}$.

(4) If $\kappa_{g}(s)^{2}>\tau_{g}(s)^{2}$, then $\boldsymbol{b}$ is a $\Delta_{3}$-dual of $\overline{\boldsymbol{D}}_{r}^{L}$.

(5) If $\tau_{g}(s)^{2}>\kappa_{g}(s)^{2}$, then $\boldsymbol{b}$ is a $\Delta_{5}$-dual of $\overline{\boldsymbol{D}}_{r}^{S}$.

Proof. We can show that (1) holds as follows:

(1) We define a mapping $\mathcal{L}_{1}: I \longrightarrow \Delta_{1}$ by $\mathcal{L}_{1}(s)=\left(\boldsymbol{n}_{\boldsymbol{\gamma}}(s), \overline{\boldsymbol{D}}_{o}^{S}(s)\right)$. Then we have $\left\langle\boldsymbol{n}_{\boldsymbol{\gamma}}(s), \overline{\boldsymbol{D}}_{o}^{S}(s)\right\rangle=$ 0 and $\mathcal{L}_{1}^{*} \theta_{11}=\left\langle\boldsymbol{n}_{\gamma}^{\prime}(s), \overline{\boldsymbol{D}}_{o}^{S}(s)\right\rangle=0$. Thus $\mathcal{L}_{1}$ is an isotropic mapping, so that $\boldsymbol{n}_{\boldsymbol{\gamma}}$ is a $\Delta_{1}$ dual of $\overline{\boldsymbol{D}}_{o}^{S}$. 
Then we define mappings

$$
\begin{array}{ll}
\mathcal{L}_{2}: I \longrightarrow \Delta_{2} & ; \quad \mathcal{L}_{2}(s)=\left(\boldsymbol{n}_{\boldsymbol{\gamma}}(s), \overline{\boldsymbol{D}}_{o}^{L}(s)\right), \\
\mathcal{L}_{3}: I \longrightarrow \Delta_{1} & ; \quad \mathcal{L}_{3}(s)=\left(\boldsymbol{b}(s), \overline{\boldsymbol{D}}_{r}^{T}(s)\right), \\
\mathcal{L}_{4}: I \longrightarrow \Delta_{3} & \left.; \quad \mathcal{L}_{3}(s)=\left(\boldsymbol{b}(s), \overline{\boldsymbol{D}}_{r}^{L}(s)\right)\right), \\
\mathcal{L}_{5}: I \longrightarrow \Delta_{5} & ; \quad \mathcal{L}_{5}(s)=\left(\boldsymbol{b}(s), \overline{\boldsymbol{D}}_{r}^{S}(s)\right) .
\end{array}
$$

Then we can show that $\mathcal{L}_{i}(i=2,3,4,5)$ are isotropic mappings. This means that $(2),(3),(4)$ and (5) hold.

\section{$5 \quad$ Height functions}

We now introduce five families of functions on $\boldsymbol{\gamma}: I \longrightarrow M \subset \mathbb{R}_{1}^{3}$ with $\left\|\boldsymbol{t}^{\prime}(s)\right\| \neq 0,\left\|\boldsymbol{b}^{\prime}(s)\right\| \neq 0$ as follows:

$$
\begin{aligned}
& H_{r}^{T}: I \times H_{+}^{2}(-1) \longrightarrow \mathbb{R} ;(s, \boldsymbol{v}) \longmapsto\langle\boldsymbol{b}(s), \boldsymbol{v}\rangle \\
& H_{r}^{S}: I \times S_{1}^{2} \longrightarrow \mathbb{R} ;(s, \boldsymbol{v}) \longmapsto\langle\boldsymbol{b}(s), \boldsymbol{v}\rangle \\
& H_{r}^{L}: I \times L C^{*} \longrightarrow \mathbb{R} ;(s, \boldsymbol{v}) \longmapsto\langle\boldsymbol{b}(s), \boldsymbol{v}\rangle-1, \\
& H_{o}^{S}: I \times S_{1}^{2} \longrightarrow \mathbb{R} ;(s, \boldsymbol{v}) \longmapsto\left\langle\boldsymbol{n}_{\boldsymbol{\gamma}}(s), \boldsymbol{v}\right\rangle, \\
& H_{o}^{L}: I \times L C^{*} \longrightarrow \mathbb{R} ;(s, \boldsymbol{v}) \longmapsto\left\langle\boldsymbol{n}_{\boldsymbol{\gamma}}(s), \boldsymbol{v}\right\rangle+1 .
\end{aligned}
$$

For any $\boldsymbol{v}$, we define $h_{r, \boldsymbol{v}}^{T}(s)=H_{r}^{T}(s, \boldsymbol{v}), h_{r, \boldsymbol{v}}^{S}(s)=H_{r}^{S}(s, \boldsymbol{v}), h_{r, \boldsymbol{v}}^{L}(s)=H_{r}^{L}(s, \boldsymbol{v}), h_{o, \boldsymbol{v}}^{S}(s)=$ $H_{o}^{S}(s, \boldsymbol{v}), h_{o, v}^{L}(s)=H_{o}^{L}(s, \boldsymbol{v})$ Then we have the following proposition.

Proposition 5.1 Let $\gamma: I \longrightarrow M$ be a unit speed curve on a spacelike surface $M \subset \mathbb{R}_{1}^{3}$ such that $\left\|\boldsymbol{t}^{\prime}(s)\right\| \neq 0,\left\|\boldsymbol{b}^{\prime}(s)\right\| \neq 0$. Then we have the following:

(A) For any $(s, \boldsymbol{v}) \in I \times H^{2}(-1)$, we have the following:

(1) $h_{r, \boldsymbol{v}}^{T}(s)=0$ if and only if there exist $\lambda, \mu \in \mathbb{R}$ with $-\lambda^{2}+\mu^{2}=1$ such that

$$
\boldsymbol{v}=\lambda \boldsymbol{t}(s)+\mu \boldsymbol{n}_{\boldsymbol{\gamma}}(s)
$$

(2) $h_{r, \boldsymbol{v}}^{T}(s)=\left(h_{r, \boldsymbol{v}}^{T}\right)^{\prime}(s)=0$ if and only if $\kappa_{g}(s)^{2}>\tau_{g}(s)^{2}$ and $\boldsymbol{v}= \pm \overline{\boldsymbol{D}}_{r}^{T}(s)$,

(3) $h_{r, \boldsymbol{v}}^{T}(s)=\left(h_{r, \boldsymbol{v}}^{T}\right)^{\prime}(s)=\left(h_{r, \boldsymbol{v}}^{T}\right)^{\prime \prime}(s)=0$ if and only if $\kappa_{g}(s)^{2}>\tau_{g}(s)^{2}, \delta_{r}^{T}(s)=0$ and $\boldsymbol{v}= \pm \overline{\boldsymbol{D}}_{r}^{T}(s)$

(4) $h_{r, \boldsymbol{v}}^{T}(s)=\left(h_{r, \boldsymbol{v}}^{T}\right)^{\prime}(s)=\left(h_{r, \boldsymbol{v}}^{T}\right)^{\prime \prime}(s)=\left(h_{r, \boldsymbol{v}}^{T}\right)^{\prime \prime \prime}(s)=0$ if and only if $\kappa_{g}(s)^{2}>\tau_{g}(s)^{2}$, $\delta_{r}^{T}(s)=0,\left(\delta_{r}^{T}\right)^{\prime}(s)=0$ and $\boldsymbol{v}= \pm \overline{\boldsymbol{D}}_{r}^{T}(s)$.

(B) For any $(s, \boldsymbol{v}) \in I \times S_{1}^{2}$, we have the following:

(1) $h_{r, \boldsymbol{v}}^{S}(s)=0$ if and only if there exist $\lambda, \mu \in \mathbb{R}$ with $-\lambda^{2}+\mu^{2}=-1$ such that

$$
\boldsymbol{v}=\lambda \boldsymbol{t}(s)+\mu \boldsymbol{n}_{\boldsymbol{\gamma}}(s)
$$


(2) $h_{r, \boldsymbol{v}}^{S}(s)=\left(h_{r, \boldsymbol{v}}^{S}\right)^{\prime}(s)=0$ if and only if $\tau_{g}(s)^{2}>\kappa_{g}(s)^{2}$ and $\boldsymbol{v}= \pm \overline{\boldsymbol{D}}_{r}^{S}(s)$,

(3) $h_{r, \boldsymbol{v}}^{S}(s)=\left(h_{r, \boldsymbol{v}}^{S}\right)^{\prime}(s)=\left(h_{r, \boldsymbol{v}}^{S}\right)^{\prime \prime}(s)=0$ if and only if $\tau_{g}(s)^{2}>\kappa_{g}(s)^{2}, \delta_{r}^{S}(s)=0$ and $\boldsymbol{v}= \pm \overline{\boldsymbol{D}}_{r}^{S}(s)$

(4) $h_{r, \boldsymbol{v}}^{S}(s)=\left(h_{r, \boldsymbol{v}}^{S}\right)^{\prime}(s)=\left(h_{r, \boldsymbol{v}}^{S}\right)^{\prime \prime}(s)=\left(h_{r, \boldsymbol{v}}^{S}\right)^{\prime \prime \prime}(s)=0$ if and only if $\tau_{g}(s)^{2}>\kappa_{g}(s)^{2}$, $\delta_{r}^{S}(s)=0,\left(\delta_{r}^{S}\right)^{\prime}(s)=0$ and $\boldsymbol{v}= \pm \overline{\boldsymbol{D}}_{r}^{S}(s)$.

(C) For any $(s, \boldsymbol{v}) \in I \times L C^{*}$, we have the following:

(1) $h_{r, v}^{L}(s)=0$ if and only if there exist $\lambda, \mu \in \mathbb{R}$ with $\lambda^{2}-\mu^{2}=-1$ such that

$$
\boldsymbol{v}=\lambda \boldsymbol{t}(s)+\mu \boldsymbol{n}_{\gamma}(s)+\boldsymbol{b}(s),
$$

(2) $h_{r, \boldsymbol{v}}^{L}(s)=\left(h_{r, \boldsymbol{v}}^{L}\right)^{\prime}(s)=0$ if and only if $\kappa_{g}(s)^{2}>\tau_{g}(s)^{2}$ and $\boldsymbol{v}= \pm \overline{\boldsymbol{D}}_{r}^{L}(s)$,

(3) $h_{r, \boldsymbol{v}}^{L}(s)=\left(h_{r, \boldsymbol{v}}^{L}\right)^{\prime}(s)=\left(h_{r, \boldsymbol{v}}^{L}\right)^{\prime \prime}(s)=0$ if and only if $\kappa_{g}(s)^{2}>\tau_{g}(s)^{2}, \delta_{r}^{L}(s)=0$ and $\boldsymbol{v}= \pm \overline{\boldsymbol{D}}_{r}^{L}(s)$

(4) $h_{r, \boldsymbol{v}}^{L}(s)=\left(h_{r, \boldsymbol{v}}^{L}\right)^{\prime}(s)=\left(h_{r, \boldsymbol{v}}^{L}\right)^{\prime \prime}(s)=\left(h_{r, \boldsymbol{v}}^{L}\right)^{\prime \prime \prime}(s)=0$ if and only if $\kappa_{g}(s)^{2}>\tau_{g}(s)^{2}$, $\delta_{r}^{L}(s)=0,\left(\delta_{r}^{L}\right)^{\prime}(s)=0$ and $\boldsymbol{v}= \pm \overline{\boldsymbol{D}}_{r}^{L}(s)$.

(D) Suppose that $\left(\kappa_{n}(s), \tau_{g}(s)\right) \neq(0,0)$. For any $(s, \boldsymbol{v}) \in I \times S_{1}^{2}$, we have the following:

(1) $h_{o, \boldsymbol{v}}^{S}(s)=0$ if and only if there exist $\lambda, \mu \in \mathbb{R}$ with $\lambda^{2}+\mu^{2}=1$ such that

$$
\boldsymbol{v}=\lambda \boldsymbol{t}(s)+\mu \boldsymbol{b}(s)
$$

(2) $h_{o, \boldsymbol{v}}^{S}(s)=\left(h_{o, \boldsymbol{v}}^{S}\right)^{\prime}(s)=0$ if and only if $\boldsymbol{v}= \pm \overline{\boldsymbol{D}}_{o}^{S}(s)$,

(3) $h_{o, \boldsymbol{v}}^{S}(s)=\left(h_{o, \boldsymbol{v}}^{S}\right)^{\prime}(s)=\left(h_{o, \boldsymbol{v}}^{S}\right)^{\prime \prime}(s)=0$ if and only if $\delta_{o}^{S}(s)=0$ and $\boldsymbol{v}= \pm \overline{\boldsymbol{D}}_{o}^{S}(s)$,

(4) $h_{o, \boldsymbol{v}}^{S}(s)=\left(h_{o, \boldsymbol{v}}^{S}\right)^{\prime}(s)=\left(h_{o, \boldsymbol{v}}^{S}\right)^{\prime \prime}(s)=\left(h_{o, \boldsymbol{v}}^{S}\right)^{\prime \prime \prime}(s)=0$ if and only if $\delta_{o}^{S}(s)=0,\left(\delta_{o}^{S}\right)^{\prime}(s)=$ 0 and $\boldsymbol{v}= \pm \overline{\boldsymbol{D}}_{o}^{S}(s)$.

(E) Suppose that $\left(\kappa_{n}(s), \tau_{g}(s)\right) \neq(0,0)$. For any $(s, \boldsymbol{v}) \in I \times L C^{*}$, we have the following:

(1) $h_{o, \boldsymbol{v}}^{L}(s)=0$ if and only if there exist $\lambda, \mu \in \mathbb{R}$ with $\lambda^{2}+\mu^{2}=1$ such that

$$
\boldsymbol{v}=\lambda \boldsymbol{t}(s)+\mu \boldsymbol{b}(s)+\boldsymbol{n}_{\gamma}(s),
$$

(2) $h_{o, \boldsymbol{v}}^{L}(s)=\left(h_{o, \boldsymbol{v}}^{L}\right)^{\prime}(s)=0$ if and only if $\boldsymbol{v}= \pm \overline{\boldsymbol{D}}_{o}^{L}(s)$,

(3) $h_{o, \boldsymbol{v}}^{L}(s)=\left(h_{o, \boldsymbol{v}}^{L}\right)^{\prime}(s)=\left(h_{o, \boldsymbol{v}}^{L}\right)^{\prime \prime}(s)=0$ if and only if $\delta_{o}^{L}(s)=0$ and $\boldsymbol{v}= \pm \overline{\boldsymbol{D}}_{o}^{L}(s)$,

(4) $h_{o, \boldsymbol{v}}^{L}(s)=\left(h_{o, \boldsymbol{v}}^{L}\right)^{\prime}(s)=\left(h_{o, \boldsymbol{v}}^{L}\right)^{\prime \prime}(s)=\left(h_{o, \boldsymbol{v}}^{L}\right)^{\prime \prime \prime}(s)=0$ if and only if $\delta_{o}^{L}(s)=0,\left(\delta_{o}^{L}\right)^{\prime}(s)=$ 0 and $\boldsymbol{v}= \pm \overline{\boldsymbol{D}}_{o}^{L}(s)$.

Proof. We remark that $\left\|\boldsymbol{t}^{\prime}(s)\right\| \neq 0,\left\|\boldsymbol{b}^{\prime}(s)\right\| \neq 0$ if and only if $-\kappa_{n}(s)^{2}+\kappa_{g}(s)^{2} \neq 0, \kappa_{g}(s)^{2}-$ $\tau_{g}(s)^{2} \neq 0$. 
(A) By straight forward calculations, we have the following :

$$
\begin{aligned}
h_{r, \boldsymbol{v}}^{T}= & \langle\boldsymbol{b}, \boldsymbol{v}\rangle \\
\left(h_{r, \boldsymbol{v}}^{T}\right)^{\prime}= & \left\langle-\kappa_{g} \boldsymbol{t}+\tau_{g} \boldsymbol{n}_{\boldsymbol{\gamma}}, \boldsymbol{v}\right\rangle \\
\left(h_{r, \boldsymbol{v}}^{T}\right)^{\prime \prime}= & \left\langle\left(-\kappa_{g}^{\prime}+\kappa_{n} \tau_{g}\right) \boldsymbol{t}-\left(\kappa_{g}^{2}-\tau_{g}^{2}\right) \boldsymbol{b}+\left(\tau_{g}^{\prime}-\kappa_{g} \kappa_{n}\right) \boldsymbol{n}_{\boldsymbol{\gamma}}, \boldsymbol{v}\right\rangle \\
\left(h_{r, \boldsymbol{v}}^{T}\right)^{\prime \prime \prime}= & \left\langle\left(-\kappa_{g}^{\prime \prime}+\kappa_{n}^{\prime} \tau_{g}+2 \kappa_{n} \tau_{g}^{\prime}+\kappa_{g}^{3}-\kappa_{g} \tau_{g}^{2}-\kappa_{n}^{2} \kappa_{g}\right) \boldsymbol{t}\right. \\
& \left.+\left(-3 \kappa_{g} \kappa_{g}^{\prime}+3 \tau_{g} \tau_{g}^{\prime}\right) \boldsymbol{b}+\left(\tau_{g}^{\prime \prime}-\kappa_{g} \kappa_{n}^{\prime}-2 \kappa_{n} \kappa_{g}^{\prime}+\tau_{g}^{3}+\kappa_{n}^{2} \tau_{g}-\kappa_{g}^{2} \tau_{g}\right) \boldsymbol{n}_{\boldsymbol{\gamma}}, \boldsymbol{v}\right\rangle .
\end{aligned}
$$

Since $\left\{\boldsymbol{t}(s), \boldsymbol{n}_{\boldsymbol{\gamma}}(s), \boldsymbol{b}(s)\right\}$ is a pseudo-orthonormal frame of $\mathbb{R}_{1}^{3}$ along $\boldsymbol{\gamma}$, we have $\boldsymbol{v}=\lambda \boldsymbol{t}(s)+$ $\mu \boldsymbol{n}_{\gamma}(s)+\eta \boldsymbol{b}(s)$.

1. Since $h_{r, \boldsymbol{v}}^{T}=0, \eta=0$, so that we have $\boldsymbol{v}=\lambda \boldsymbol{t}(s)+\mu \boldsymbol{n}_{\boldsymbol{\gamma}}(s)$. Here, $\boldsymbol{v}$ is timelike. Then we have $\mu^{2}>\lambda^{2}$. Thus, $\mu \neq 0$. This completes the proof of assertion (A),(1).

2. Moreover, $\left(h_{r, \boldsymbol{v}}^{T}\right)^{\prime}=0$ implies $-\lambda \kappa_{g}-\mu \tau_{g}=0$. Therefore, we have $\kappa_{g} \boldsymbol{v}=\kappa_{g} \lambda \boldsymbol{t}+\kappa_{g} \mu \boldsymbol{n}_{\boldsymbol{\gamma}}=$ $-\mu\left(\tau_{g} \boldsymbol{t}-\kappa_{g} \boldsymbol{n}_{\gamma}\right)$. Thus we have $-\kappa_{g}^{2}=\mu^{2}\left(\tau_{g}^{2}-\kappa_{g}^{2}\right)$, so that $\tau_{g}^{2} \leq \kappa_{g}^{2}$. Since $\kappa_{g}(s)^{2}-\tau_{g}(s)^{2} \neq 0$, we have $\tau_{g}^{2}<\kappa_{g}^{2}$. It follows that

$$
\boldsymbol{v}= \pm \frac{\tau_{g} \boldsymbol{t}-\kappa_{g} \boldsymbol{n}_{\gamma}}{\sqrt{\kappa_{g}^{2}-\tau_{g}^{2}}}= \pm \overline{\boldsymbol{D}}_{r}^{T}
$$

3. If we add extra condition $\left(h_{r, \boldsymbol{v}}^{T}\right)^{\prime \prime}=0$, then we have

$$
\kappa_{n}-\frac{\kappa_{g} \tau_{g}^{\prime}-\kappa_{g}^{\prime} \tau_{g}}{\kappa_{g}^{2}-\tau_{g}^{2}}=0
$$

Thus we have $\delta_{r}^{T}=0$.

4. Moreover, if we consider one more condition $\left(h_{r, \boldsymbol{v}}^{T}\right)^{\prime \prime \prime}=0$, then we have

$$
\kappa_{n}^{\prime}\left(\kappa_{g}^{2}-\tau_{g}^{2}\right)+2\left(\kappa_{g} \kappa_{g}^{\prime}-\tau_{g} \tau_{g}^{\prime}\right)\left(\delta_{r}^{T}+\frac{\kappa_{g} \tau_{g}^{\prime}-\kappa_{g}^{\prime} \tau_{g}}{\kappa_{g}^{2}-\tau_{g}^{2}}\right)-\left(\kappa_{g} \tau_{g}^{\prime \prime}-\kappa_{g}^{\prime \prime} \tau_{g}\right)=0 .
$$

Since we have

$$
\left(\delta_{r}^{T}\right)^{\prime}=\kappa_{n}^{\prime}+\frac{2\left(\kappa_{g} \kappa_{g}^{\prime}-\tau_{g} \tau_{g}^{\prime}\right)\left(\kappa_{g} \tau_{g}^{\prime}-\kappa_{g}^{\prime} \tau_{g}\right)}{\left(\kappa_{g}^{2}-\tau_{g}^{2}\right)^{2}}-\frac{\left(\kappa_{g} \tau_{g}^{\prime \prime}-\kappa_{g}^{\prime \prime} \tau_{g}\right)}{\left(\kappa_{g}^{2}-\tau_{g}^{2}\right)},
$$

$\left(\delta_{r}^{T}\right)^{\prime}=0$ with the condition $\delta_{r}^{T}=0$.

For other cases (B), (C), (D) and (E), we have the similar calculations to case (A) for the derivatives of $h_{r, \boldsymbol{v}}^{S}, h_{r, \boldsymbol{v}}^{L}, h_{o, \boldsymbol{v}}^{S}$ and $h_{o, \boldsymbol{v}}^{L}$, respectively. We omit the details here. 


\section{$6 \quad$ Proof of Theorem 3.1}

In this section we give a proof of Theorem 3.1. In order to prove Theorem 3.1, we use some general results on the singularity theory for families of function germs. Detailed descriptions are found in the book $\left[3\right.$. Let $F:\left(\mathbb{R} \times \mathbb{R}^{r},\left(s_{0}, x_{0}\right)\right) \longrightarrow \mathbb{R}$ be a function germ. We call $F$ an $r$-parameter unfolding of $f$, where $f(s)=F_{x_{0}}\left(s, x_{0}\right)$. We say that $f$ has an $A_{k}$-singularity at $s_{0}$ if $f^{(p)}\left(s_{0}\right)=0$ for all $1 \leq p \leq k$, and $f^{(k+1)}\left(s_{0}\right) \neq 0$. We also say that $f$ has an $A_{\geq k^{-}}$ singularity at $s_{0}$ if $f^{(p)}\left(s_{0}\right)=0$ for all $1 \leq p \leq k$. Let $F$ be an unfolding of $f$ and $f(s)$ has an $A_{k}$-singularity $(k \geq 1)$ at $s_{0}$. We denote the $(k-1)$-jet of the partial derivative $\frac{\partial F}{\partial x_{i}}$ at $s_{0}$ by $j^{(k-1)}\left(\frac{\partial F}{\partial x_{i}}\left(s, x_{0}\right)\right)\left(s_{0}\right)=\sum_{j=0}^{k-1} \alpha_{j i}\left(s-s_{0}\right)^{j}$ for $i=1, \ldots, r$. Then $F$ is called an $\mathcal{R}$-versal unfolding if the $k \times r$ matrix of coefficients $\left(\alpha_{j i}\right)_{j=0, \ldots, k-1 ; i=1, \ldots, r}$ has rank $k(k \leq r)$. We introduce an important set concerning the unfoldings relative to the above notions. The discriminant set of $F$ is the set

$$
\mathcal{D}_{F}=\left\{x \in \mathbb{R}^{r} \mid \text { there exists } s \text { with } F=\frac{\partial F}{\partial s}=0 \text { at }(s, x)\right\}
$$

Then we have the following classification (cf., 3]).

Theorem 6.1 Let $F:\left(\mathbb{R} \times \mathbb{R}^{r},\left(s_{0}, x_{0}\right)\right) \longrightarrow \mathbb{R}$ be an r-parameter unfolding of $f(s)$ which has the $A_{2}$ singularity at $s_{0}$. If $F$ is an $\mathcal{R}$-versal unfolding, then $\mathcal{D}_{F}$ is locally diffeomorphic to $C \times \mathbb{R}^{r-1}$.

Here, $C=\left\{\left(x_{1}, x_{2}\right) \mid x_{1}=t^{2}, x_{2}=t^{3}\right\}$ is the ordinary cusp (i.e. the semi-cubic parabola).

We now consider that $H_{r}^{T}, H_{r}^{S}, H_{r}^{L}, H_{o}^{T}$, and $H_{o}^{L}$ are unfoldings of $h_{r, \boldsymbol{v}}^{T}(s), h_{r, \boldsymbol{v}}^{S}(s), h_{r, \boldsymbol{v}}^{L}(s), h_{o, \boldsymbol{v}}^{T}(s)$, and $h_{o, \boldsymbol{v}}^{L}(s)$ for any $\boldsymbol{v}$, respectively.

Proposition 6.2 Let $\gamma: I \longrightarrow M$ be a unit speed curve on a spacelike surface $M \subset \mathbb{R}_{1}^{3}$ such that $\left\|\boldsymbol{t}^{\prime}(s)\right\| \neq 0,\left\|\boldsymbol{b}^{\prime}(s)\right\| \neq 0$. Then we have the following:

(A) if $h_{r, \boldsymbol{v}}^{T}$ has the $A_{2}$-singularity at $s_{0}$, then $H_{r}^{T}$ is an $\mathcal{R}$-versal unfolding of $h_{r, \boldsymbol{v}}^{T}$,

(B) if $h_{r, \boldsymbol{v}}^{S}$ has the $A_{2}$-singularity at $s_{0}$, then $H_{r}^{S}$ is an $\mathcal{R}$-versal unfolding of $h_{r, \boldsymbol{v}}^{S}$,

(C) if $h_{r, \boldsymbol{v}}^{L}$ has the $A_{2}$-singularity at $s_{0}$, then $H_{r}^{L}$ is an $\mathcal{R}$-versal unfolding of $h_{r, \boldsymbol{v}}^{L}$,

(D) if $h_{o, \boldsymbol{v}}^{S}$ has the $A_{2}$-singularity at $s_{0}$, then $H_{o}^{S}$ is an $\mathcal{R}$-versal unfolding of $h_{o, \boldsymbol{v}}^{S}$,

(E) if $h_{o}^{L}, \boldsymbol{v}$ has the $A_{2}$-singularity at $s_{0}$, then $H_{o}^{L}$ is an $\mathcal{R}$-versal unfolding of $h_{o}^{L}, \boldsymbol{v}$.

Proof. Here, we only give the proof for (A). Other cases are similar to case (A).

(A) We denote that $\boldsymbol{v}=\left(\sqrt{x_{1}^{2}+x_{2}^{2}+1}, x_{1}, x_{2}\right) \in H_{+}^{2}(-1), \boldsymbol{b}=\left(b_{0}(s), b_{1}(s), b_{2}(s)\right)$. Then we have

$$
H_{r}^{T}(s, \boldsymbol{v})=-b_{0}(s) \sqrt{x_{1}^{2}+x_{2}^{2}+1}+b_{1}(s) x_{1}+b_{2}(s) x_{2}
$$

and

$$
\frac{\partial H_{r}^{T}}{\partial x_{1}}(s, \boldsymbol{v})=-b_{0}(s) \frac{x_{1}}{\sqrt{x_{1}^{2}+x_{2}^{2}+1}}+b_{1}(s), \frac{\partial H_{r}^{T}}{\partial x_{1}}(s, \boldsymbol{v})=-b_{0}(s) \frac{x_{2}}{\sqrt{x_{1}^{2}+x_{2}^{2}+1}}+b_{2}(s) .
$$


Therefore the 2-jet of $H_{r}^{T}(s, \boldsymbol{v})$ are

$$
\begin{aligned}
j^{2} \frac{\partial H_{r}^{T}}{\partial x_{1}}\left(s_{0}, \boldsymbol{v}\right)= & \left(-b_{0}\left(s_{0}\right) \frac{x_{1}}{\sqrt{x_{1}^{2}+x_{2}^{2}+1}}+b_{1}\left(s_{0}\right)\right) \\
& +\left(-b_{0}^{\prime}\left(s_{0}\right) \frac{x_{1}}{\sqrt{x_{1}^{2}+x_{2}^{2}+1}}+b_{1}^{\prime}\left(s_{0}\right)\right)\left(s-s_{0}\right), \\
j^{2} \frac{\partial H_{r}^{T}}{\partial x_{2}}\left(s_{0}, \boldsymbol{v}\right)= & \left(-b_{0}\left(s_{0}\right) \frac{x_{2}}{\sqrt{x_{1}^{2}+x_{2}^{2}+1}}+b_{2}\left(s_{0}\right)\right) \\
& +\left(-b_{0}^{\prime}\left(s_{0}\right) \frac{x_{2}}{\sqrt{x_{1}^{2}+x_{2}^{2}+1}}+b_{2}^{\prime}\left(s_{0}\right)\right)\left(s-s_{0}\right) .
\end{aligned}
$$

We consider the following matrix:

$$
A=\left(\begin{array}{ll}
-b_{0}\left(s_{0}\right) \frac{x_{1}}{\sqrt{x_{1}^{2}+x_{2}^{2}+1}}+b_{1}\left(s_{0}\right) & -b_{0}\left(s_{0}\right) \frac{x_{2}}{\sqrt{x_{1}^{2}+x_{2}^{2}+1}}+b_{2}\left(s_{0}\right) \\
-b_{0}^{\prime}\left(s_{0}\right) \frac{x_{1}}{\sqrt{x_{1}^{2}+x_{2}^{2}+1}}+b_{1}^{\prime}\left(s_{0}\right) & -b_{0}^{\prime}\left(s_{0}\right) \frac{x_{2}}{\sqrt{x_{1}^{2}+x_{2}^{2}+1}}+b_{2}^{\prime}\left(s_{0}\right)
\end{array}\right)
$$

The determinant of $A$ is

$$
\begin{aligned}
\operatorname{det} A & =\frac{1}{\sqrt{x_{1}^{2}+x_{2}^{2}+1}}\left(x_{1}\left(b_{0}^{\prime} b_{2}-b_{0} b_{2}^{\prime}\right)+x_{2}\left(b_{0} b_{1}^{\prime}-b_{0}^{\prime} b_{1}\right)+\sqrt{x_{1}^{2}+x_{2}^{2}+1}\left(b_{2}^{\prime} b_{1}-b_{2} b_{1}^{\prime}\right)\right) \\
& =\frac{1}{\sqrt{x_{1}^{2}+x_{2}^{2}+1}}\left\langle\left(b_{2} b_{1}^{\prime}-b_{1} b_{2}^{\prime}, b_{2} b_{0}^{\prime}-b_{2}^{\prime} b_{0}, b_{0} b_{1}^{\prime}-b_{0}^{\prime} b_{1}\right),\left(\sqrt{x_{1}^{2}+x_{2}^{2}+1}, x_{1}, x_{2}\right)\right\rangle \\
& =\frac{1}{\sqrt{x_{1}^{2}+x_{2}^{2}+1}}\left\langle\left(\boldsymbol{b} \wedge \boldsymbol{b}^{\prime}\right), \boldsymbol{v}\right\rangle \\
& =\frac{1}{\sqrt{x_{1}^{2}+x_{2}^{2}+1}}\left|\boldsymbol{b} \boldsymbol{b}^{\prime} \boldsymbol{v}\right|
\end{aligned}
$$

By Proposition 5.1, if $h_{r, \boldsymbol{v}}^{T}$ has the $A_{2}$-singularity at $s_{0}$, then $\boldsymbol{v}= \pm \overline{\boldsymbol{D}}_{r}^{T}(s)$ and $\boldsymbol{b}^{\prime}(s)=$ $-\kappa_{g}(s) \boldsymbol{t}(s)+\tau_{g}(s) \boldsymbol{n}_{\boldsymbol{\gamma}}(s)$, so that $\left\{\boldsymbol{b}, \boldsymbol{b}^{\prime}, \boldsymbol{v}\right\}$ is linearly independent. Therefore, $\operatorname{rank} A=2$. This means that $H_{r}^{T}$ is an $\mathcal{R}$-versal unfolding of $h_{r, \boldsymbol{v}}^{T}$.

We define three vector fields respectively defined as normalizations of $\boldsymbol{t}^{\prime}, \boldsymbol{n}_{\gamma}^{\prime}, \boldsymbol{b}^{\prime}$ as follows:

$\boldsymbol{T}_{\boldsymbol{t}}(s)=\frac{\kappa_{n}(s) \boldsymbol{n}_{\boldsymbol{\gamma}}(s)+\kappa_{g}(s) \boldsymbol{b}(s)}{\sqrt{\kappa_{g}(s)^{2}-\kappa_{n}(s)^{2}}}, \boldsymbol{T}_{\boldsymbol{n}_{\gamma}}(s)=\frac{\kappa_{n}(s) \boldsymbol{t}(s)+\tau_{g}(s) \boldsymbol{b}(s)}{\sqrt{\kappa_{g}(s)^{2}+\tau_{g}(s)^{2}}}, \boldsymbol{T}_{\boldsymbol{b}}(s)=\frac{-\kappa_{g}(s) \boldsymbol{t}(s)+\tau_{g}(s) \boldsymbol{n}_{\boldsymbol{\gamma}}(s)}{\sqrt{\kappa_{g}(s)^{2}-\tau_{g}(s)^{2}}}$

We can prove Theorem 3.1.

Proof of Theorem 3.1. Here we only give the proof for (A) again.

(A) (1) By a straight forward calculation $\overline{\boldsymbol{D}}_{r}^{T}(s)$, we have

$$
\left(\overline{\boldsymbol{D}}_{r}^{T}(s)\right)^{\prime}=\delta_{r}^{T}(s) \boldsymbol{T}_{\boldsymbol{b}}(s)
$$

so that $\overline{\boldsymbol{D}}_{r}^{T}(s)$ is non-singular at $s=s_{0}$ if and only if $\delta_{r}^{T}\left(s_{0}\right) \neq 0$. 
(2) By Proposition 5.1, $h_{r, \boldsymbol{v}}^{T}$ is an $A_{2}$-singularity if and only if $\delta_{r}^{T}\left(s_{0}\right)=0,\left(\delta_{r}^{T}\right)^{\prime}\left(s_{0}\right) \neq 0$ and $\boldsymbol{v}=\overline{\boldsymbol{D}}_{r}^{T}(s)$. By Proposition 6.2, $H_{r}^{T}$ is an $\mathcal{R}$-versal unfolding of $h_{r, \boldsymbol{v}}^{T}$. By Proposition 5.1, the image of $\overline{\boldsymbol{D}}_{r}^{T}$ is the discriminant set of $H_{r}^{T}$. By Theorem 6.1, the discriminant of $H_{r}^{T}$ is locally diffeomorphic to the cusp $C$.

\section{$7 \quad$ Invariants of curves on surfaces}

In this section we consider geometric meanings of the invariants $\delta_{r}^{T}, \delta_{r}^{S}, \delta_{r}^{L}, \delta_{o}^{S}, \delta_{o}^{L}$. In particular what can we say about the original curve on the surface when each invariant is vanishing. For the purpose, we consider cylinders in Lorentz-Minkowski space. A (generalized) cylinder in $\mathbb{R}_{1}^{3}$ is a ruled surface with a constant director. It is parametrized by $F(t, u)=\boldsymbol{\sigma}(t)+u \boldsymbol{v}$, where $\boldsymbol{\sigma}$ is a smooth curve and $\boldsymbol{v}$ is a non-zero vector. The vector $\boldsymbol{v}$ is called the director. We say that $F$ is a spacelike cylinder, a timelike cylinder and a lightlike cylinder if the director $\boldsymbol{v}$ is spacelike, timelike and lightlike, respectively. Let $M$ be a surface and $N$ be a cylinder in $\mathbb{R}_{1}^{3}$. We say that $N$ is a pseudo-normal cylinder of $M$ if $M \cap N \neq \emptyset$ and $T_{p} N$ contains the pseudo-normal vector $\boldsymbol{n}(p)$ at any $p \in M \cap N$. In this case $M$ and $N$ transversally intersect, so that $M \cap N$ is a regular curve $C$. We call $C$ a slice of $M$ with a pseudo-normal cylinder of $M$. Moreover, we call $N$ a pseudo-normal spacelike cylinder if the director of $N$ is spacelike and a pseudo-normal timelike cylinder if the director of $N$ is timelike, respectively. We remark that the director of $N$ is not lightlike. If $N$ is locally parametrized by $F(t, u)=\boldsymbol{\sigma}(t)+u \boldsymbol{v}$, then we have

$$
\frac{\partial F}{\partial t}(t, u)=\boldsymbol{\sigma}^{\prime}(t) \text { and } \frac{\partial F}{\partial u}(t, u)=\boldsymbol{v},
$$

so that the pseudo-normal to $N$ is given by

$$
\frac{\partial F}{\partial t}(t, u) \times \frac{\partial F}{\partial u}(t, u)=\boldsymbol{\sigma}^{\prime}(t) \times \boldsymbol{v} .
$$

If $C$ is parametrized by $\gamma(s)$, where $s$ is the arc-length parameter of $\gamma$, then $N$ can be parameterized by $F(s, u)=\gamma(s)+u \boldsymbol{v}$ at least locally. Since $N$ has been given an orientation by $F$, the unit normal vector of $N$ along $C$ is $\boldsymbol{b}(s)$. In particular, $\langle\boldsymbol{b}(s), \boldsymbol{v}\rangle=0$.

On the other hand, $N$ is called a osculating cylinder if the tangent planes of $M$ and $N$ coincide at any point of $M \cap N$. In this case $C=M \cap N$ is called a slice of $M$ with an osculating cylinder of $M$. We remark that the director of the osculating cylinder is always spacelike. If $N$ is locally parametrized by $F(t, u)=\boldsymbol{\sigma}(t)+u \boldsymbol{v}$, then the unit normal vector of $N$ along $C$ is $\boldsymbol{n}_{\boldsymbol{\gamma}}$ and $\langle\boldsymbol{n}, \boldsymbol{v}\rangle=0$ for a parmetrization $\boldsymbol{\gamma}$ of $C$.

We call $N$ a hyperbolic lightlike cylinder if $M \cap N \neq \emptyset$ and $\langle\boldsymbol{n}(p), \boldsymbol{v}\rangle=-1$ at any point $p \in M \cap N$, where $\boldsymbol{v}$ is the lightlike director of $N$. In this case, $N$ is transversely intersect with $M$, so that $C=M \cap N$ is a regular curve. We call $C$ a slice of $M$ with a hyperbolic lightlike cylinder. We also call $N$ a de Sitter lightlike cylinder if $M \cap N \neq \emptyset$ and $\langle\boldsymbol{b}(p), \boldsymbol{v}\rangle=1$ at any point $p \in M \cap N$, where $\boldsymbol{v}$ is the lightlike director of $N$. In this case, $N$ is transversely intersect with $M$, so that $C=M \cap N$ is a regular curve. We call $C$ a slice of $M$ with a de Sitter lightlike cylinder. For the both cases in the above, $\boldsymbol{v}$ is lightlike.

Then we have the following theorem.

Theorem 7.1 Let $\gamma: I \longrightarrow M$ be a unit speed curve on a spacelike surface $M \subset \mathbb{R}_{1}^{3}$ such that $\left\|\boldsymbol{t}^{\prime}(s)\right\| \neq 0,\left\|\boldsymbol{b}^{\prime}(s)\right\| \neq 0$. 
(A) Suppose that $\kappa_{g}(s)^{2}>\tau_{g}(s)^{2}$. Then the following conditions are equivalent:

(1) $\overline{\boldsymbol{D}}_{r}^{T}(s)$ is a constant vector,

(2) $\delta_{r}^{T}(s) \equiv 0$,

(3) $\gamma(I)$ is the slice of $M$ with a timelike pseudo-normal cylinder,

(4) $\boldsymbol{b}(I)$ is a subset of a hyperbolic line in $H^{2}(-1)$.

(B) Suppose that $\tau_{g}(s)^{2}>\kappa_{g}(s)^{2}$. Then the following conditions are equivalent:

(1) $\overline{\boldsymbol{D}}_{r}^{S}(s)$ is a constant vector,

(2) $\delta_{r}^{S}(s) \equiv 0$,

(3) $\gamma(I)$ is the slice of $M$ with a spacelike pseudo-normal cylinder,

(4) $\boldsymbol{b}(I)$ is a subset of a geodesic pseudo-circle in $S_{1}^{2}$.

(C) Suppose that $\kappa_{g}(s)^{2}>\tau_{g}(s)^{2}$. Then the following conditions are equivalent:

(1) $\overline{\boldsymbol{D}}_{r}^{L}(s)$ is a constant vector,

(2) $\delta_{r}^{L}(s) \equiv 0$,

(3) $\gamma(I)$ is the slice of $M$ with a de Sitter lightlike cylinder,

(4) $\boldsymbol{b}(I)$ is a subset of de Sitter horocycle in $S_{1}^{2}$.

(D) Suppose that $\left(\kappa_{n}(s), \tau_{g}(s)\right) \neq(0,0)$. Then the following conditions are equivalent:

(1) $\overline{\boldsymbol{D}}_{o}^{S}(s)$ is a constant vector,

(2) $\delta_{o}^{S}(s) \equiv 0$,

(3) $\gamma(I)$ is the slice of $M$ with a pseudo-osculating cylinder,

(4) $\boldsymbol{n}_{\boldsymbol{\gamma}}(I)$ is a subset of a geodesic hyperbola in $S_{1}^{2}$.

(E) Suppose that $\left(\kappa_{n}(s), \tau_{g}(s)\right) \neq(0,0)$. Then the following conditions are equivalent:

(1) $\overline{\boldsymbol{D}}_{o}^{L}(s)$ is a constant vector,

(2) $\delta_{o}^{L}(s) \equiv 0$,

(3) $\gamma(I)$ is the slice of $M$ with a hyperbolic lightlike cylinder,

(4) $\boldsymbol{n}_{\boldsymbol{\gamma}}(I)$ is a subset of a horocycle in $H^{2}(-1)$.

Proof. The proof of (B) and (D) are similar to the proof of (A) Moreover, the proof of (E) is similar to the proof of $(\mathrm{C})$. Therefore, we only give the proof of $(\mathrm{A})$ and $(\mathrm{C})$.

(A) Since $\left(\overline{\boldsymbol{D}}_{r}^{T}(s)\right)^{\prime}=\delta_{r}^{T}(s) \boldsymbol{T}_{\boldsymbol{b}}(s)$, conditions (1) and (2) are equivalent. Suppose that (3) holds. Then there exists $\boldsymbol{v} \in H_{+}^{2}(-1)$ such that $\langle\boldsymbol{b}(s), \boldsymbol{v}\rangle \equiv 0$. Thus, there exist $\lambda, \mu \in \mathbb{R}$ such that $\boldsymbol{v}=\lambda \boldsymbol{t}(s)+\mu \boldsymbol{n}_{\boldsymbol{\gamma}}(s)$. Since $\langle\boldsymbol{b}(s), \boldsymbol{v}\rangle \equiv 0$, we have $\left\langle\boldsymbol{b}^{\prime}(s), \boldsymbol{v}\right\rangle \equiv 0$. It follows that $-\lambda \kappa_{g}(s)-\mu \tau_{g}(s)=0$. Then $\boldsymbol{v}=\overline{\boldsymbol{D}}_{r}^{T}(s)$. This means that (1) holds. If (1) holds, then $\overline{\boldsymbol{D}}_{r}^{T}(s)$ is a constant vector $\boldsymbol{v}=\overline{\boldsymbol{D}}_{r}^{T}(s) \in H_{+}^{2}(-1)$. Since we have $\langle\boldsymbol{b}(s), \boldsymbol{v}\rangle=$ 
$\left\langle\boldsymbol{b}(s), \overline{D_{r}^{T}}(s)\right\rangle=0,(3)$ holds. Moreover, the above equality means that $\boldsymbol{b}(s) \in P(\boldsymbol{v}, 0)$. This means that the image of $\boldsymbol{b}$ is a subset of the hyperbolic line $P(\boldsymbol{v}, 0) \cap H^{2}(-1)$. Thus (4) holds. For the converse, there exists $\boldsymbol{v} \in H^{2}(-1)$ such that $\langle\boldsymbol{b}(s), \boldsymbol{v}\rangle=0$. Then there exist $\lambda, \mu \in \mathbb{R}$ such that $\boldsymbol{v}=\lambda \boldsymbol{t}(s)+\mu \boldsymbol{n}_{\boldsymbol{\gamma}}(s)$. Since $\langle\boldsymbol{b}(s), \boldsymbol{v}\rangle=0,\left\langle\boldsymbol{b}^{\prime}(s), \boldsymbol{v}\right\rangle=0$, so that we have $-\lambda \kappa_{g}(s)-\mu \tau_{g}(s)=0$. Therefore, $\boldsymbol{v}=\overline{\boldsymbol{D}}_{r}^{T}(s)$. Thus (1) holds.

(C) Since $\left(\overline{D_{r}^{L}}(s)\right)^{\prime}=\delta_{r}^{L}(s) \boldsymbol{T}_{\boldsymbol{b}}(s)$, (1) and (2) are equivalent. Suppose that (3) holds. Then there exists $\boldsymbol{v} \in L C^{*}$ such that $\langle\boldsymbol{b}(s), \boldsymbol{v}\rangle=1$. We put $\boldsymbol{\alpha}=\boldsymbol{v}-\boldsymbol{b}(s)$. It follows that $\langle\boldsymbol{\alpha}, \boldsymbol{\alpha}\rangle=-1$, so that $\boldsymbol{\alpha} \in H^{2}(-1)$. Moreover, we have $\langle\boldsymbol{b}(s), \boldsymbol{\alpha}\rangle=0,\left\langle\boldsymbol{b}^{\prime}(s), \boldsymbol{\alpha}\right\rangle=0$. This means that $\boldsymbol{\alpha}$ is a $\Delta_{1}$-dual of $\boldsymbol{b}$. By the similar arguments to $(\mathrm{A})$, we have $\overline{D_{r}^{T}}(s)=$ $\boldsymbol{v}-\boldsymbol{b}(s)$, so that $\boldsymbol{v}=\overline{D_{r}^{T}}(s)+\boldsymbol{b}(s)=\overline{D_{r}^{L}}(s)$. Thus (1) holds. For the converse, if (1) holds, then we have $\left\langle\boldsymbol{b}(s), \underline{\boldsymbol{v}\rangle}=1\right.$ for $\boldsymbol{v}=\overline{D_{r}^{L}}(s)$. Therefore, (3) holds. Moreover, if (1) holds, then we have $\left\langle\boldsymbol{b}(s), \overline{D_{r}^{L}}(s)\right\rangle=1$, so that the image of $\boldsymbol{b}$ is a subset of a de Sitter horocycle. For the converse, suppose that (4) holds. Then there exists $\boldsymbol{v} \in L C^{*}$ such that $\langle\boldsymbol{b}(s), \boldsymbol{v}\rangle=-1$. There exist $\lambda, \mu \in \mathbb{R}$ such that $\boldsymbol{v}=\lambda \boldsymbol{t}(s)+\mu \boldsymbol{n}_{\boldsymbol{\gamma}}(s)$. Since $\langle\boldsymbol{b}(s), \boldsymbol{v}\rangle=-1$, we have $\left\langle\boldsymbol{b}^{\prime}(s), \boldsymbol{v}\right\rangle=0$. This means that $-\lambda \kappa_{g}(s)-\mu \tau_{g}(s)=0$, so that we have $\boldsymbol{v}=\overline{D_{r}^{L}}(s)$. Thus (1) holds.

This completes the proof.

\section{Examples}

In this section we consider some examples.

\subsection{Spacelike planes}

We now consider that $M=\mathbb{R}_{0}^{2}=\left\{\boldsymbol{x}=\left(x_{0}, x_{1}, x_{2}\right) \in \mathbb{R}_{1}^{3} \mid x_{0}=0\right\}$. Then we have a unit speed curve $\gamma: I \longrightarrow \mathbb{R}_{0}^{2}$, which can be considered as a curve on the Euclidean plane. In this case we have $\boldsymbol{n}_{\gamma}(s)=\boldsymbol{e}_{0}, \boldsymbol{t}(s)=\boldsymbol{\gamma}^{\prime}(s), \boldsymbol{b}(s)=\boldsymbol{e}_{0} \wedge \boldsymbol{t}(s)$. Since $\boldsymbol{n}_{\gamma}^{\prime}=\boldsymbol{e}_{0}^{\prime} \equiv 0$, we have $\kappa_{n}(s) \equiv \tau_{g}(s) \equiv 0$, so that

$$
\left\{\begin{array}{l}
\boldsymbol{t}^{\prime}(s)=\kappa(s) \boldsymbol{b}(s), \\
\boldsymbol{b}^{\prime}(s)=-\kappa(s) \boldsymbol{t}(s),
\end{array}\right.
$$

where $\kappa(s)=\kappa_{g}(s)=\left\langle\boldsymbol{t}^{\prime}(s), \boldsymbol{b}(s)\right\rangle$ is the curvature of $\boldsymbol{\gamma}$ as a Euclidean plane curve. In this case the Lorentzian Darboux vectors are $\overline{\boldsymbol{D}}_{r}^{T}(s)=\mp \boldsymbol{n}_{\gamma}(s)=\mp \boldsymbol{e}_{0}, \overline{\boldsymbol{D}}_{r}^{L}(s)=\mp \boldsymbol{n}_{\gamma}(s)+\boldsymbol{b}(s)=$ $\mp \boldsymbol{e}_{0}+\boldsymbol{b}(s), \overline{\boldsymbol{D}}_{o}^{S}(s)=\mp \boldsymbol{b}(s)$ and $\overline{\boldsymbol{D}}_{o}^{L}(s)=\mp \boldsymbol{b}(s)+\boldsymbol{e}_{0}$. Here, $\overline{\boldsymbol{D}}_{r}^{S}(s)$ is not well-defined. Thus, $\overline{\boldsymbol{D}}_{o}^{S}, \overline{\boldsymbol{D}}_{o}^{L}$ and $\overline{\boldsymbol{D}}_{r}^{L}$ correspond to the ordinary Gauss map of the curve as a Euclidean plane curve. Moreover, we have $\delta_{r}^{T}(s) \equiv 0, \delta_{r}^{L}(s)=\mp \kappa_{g}(s)=\mp \kappa(s)$ and $\delta_{o}^{S}(s)=\delta_{o}^{L}(s)=\kappa_{g}(s)=\kappa(s)$.

\subsection{The hyperbolic plane}

We consider that $M=H^{2}(-1)$. For a unit speed curve $\gamma: I \longrightarrow H^{2}(-1)$, we can take $\boldsymbol{n}_{\boldsymbol{\gamma}}(s)=\boldsymbol{\gamma}(s), \boldsymbol{t}(s)=\boldsymbol{\gamma}^{\prime}(s)$. Then we have the Lorentzian Darboux frame $\{\boldsymbol{t}, \boldsymbol{\gamma}, \boldsymbol{b}\}$, which is called a Lorentzian Sabban frame. In this case we have $\kappa_{n}(s) \equiv 1$ and $\tau_{g}(s) \equiv 0$. Thus the 
Therefore, we have

$$
\left\{\begin{array}{l}
\boldsymbol{t}^{\prime}(s)=\gamma(s)+\kappa_{g}(s) \boldsymbol{b}(s), \\
\gamma^{\prime}(s)=\boldsymbol{t}(s) \\
\boldsymbol{b}^{\prime}(s)=-\kappa_{g}(s) \boldsymbol{t}(s)
\end{array}\right.
$$

In this case the Lorentzian Darboux vectors are $\overline{\boldsymbol{D}}_{r}^{T}(s)=\mp \boldsymbol{\gamma}(s), \overline{\boldsymbol{D}}_{r}^{L}(s)=\mp \boldsymbol{\gamma}(s)+\boldsymbol{b}(s)$, $\overline{\boldsymbol{D}}_{o}^{S}(s)=\mp \boldsymbol{b}(s)$ and $\overline{\boldsymbol{D}}_{o}^{L}(s)=\gamma(s) \mp \boldsymbol{b}(s)$. Here $\overline{\boldsymbol{D}}_{r}^{S}(s)$ is not well-defined. It follows that $\delta_{r}^{T}(s)=1, \delta_{r}^{L}(s)=1 \pm \kappa_{g}(s), \delta_{o}^{S}(s)=\kappa_{g}(s)$, and $\delta_{o}^{L}(s)=\kappa_{g}(s) \pm 1$. We remark that $\overline{\boldsymbol{D}}_{r}^{L}=\mp \overline{\boldsymbol{D}}_{o}^{L}$ are called hyperbolic Gauss indicatrices in [4].

\subsection{Spacelike developable surfaces}

We consider an spacelike embedding $\boldsymbol{X}(x, y)=\left(\sqrt{x^{2}+1}, x, y\right)$ and $M=\boldsymbol{X}\left(\mathbb{R}^{2}\right)$. By straight forward calculations, we have $\boldsymbol{n}(x, y)=\left(-\sqrt{x^{2}+1},-x, 0\right)$. We now consider a curve on $M$ defined by $\gamma(s)=\left(\sqrt{s^{2}+1}, s, f(s)\right)$. Then $\gamma^{\prime}(s)=\left(\frac{s}{\sqrt{s^{2}+1}}, 1, f^{\prime}(s)\right)$. Here $s$ is an arc-length parameter if and only if $f^{\prime}(s)^{2}\left(s^{2}+1\right)=s^{2}$. With this condition, $\boldsymbol{t}(s)=\boldsymbol{\gamma}^{\prime}(s)$ is the unit tangent vector of $\boldsymbol{\gamma}$. Then we have $\boldsymbol{n}_{\boldsymbol{\gamma}}(s)=\left(-\sqrt{s^{2}+1},-s, 0\right)$ and

$$
\boldsymbol{b}(s)=\left(-s f^{\prime}(s),-f^{\prime}(s) \sqrt{s^{2}+1}, \frac{1}{\sqrt{s^{2}+1}}\right) .
$$

It follows that

$$
\kappa_{g}(s)=\frac{s f^{\prime}(s)+f^{\prime \prime}(s)\left(s^{2}+1\right)}{\left(s^{2}+1\right)^{3 / 2}}, \kappa_{n}(s)=\frac{-1}{s^{2}+1}, \tau_{g}(s)=\frac{f^{\prime}(s)}{\sqrt{s^{2}+1}} .
$$

Then

$$
\left(\tau_{g} \boldsymbol{t}-\kappa_{g} \boldsymbol{n}_{\boldsymbol{\gamma}}\right)(s)=\left(\frac{2 s f^{\prime}(s)+f^{\prime \prime}(s)\left(s^{2}+1\right)}{s^{2}+1}, \frac{f^{\prime}(s)\left(s^{2}+1\right)+s f^{\prime}(s)+f^{\prime \prime}(s)\left(s^{2}+1\right)}{\left(s^{2}+1\right)^{3 / 2}}, \frac{f^{\prime \prime}(s)}{\sqrt{s^{2}+1}}\right) .
$$

Moreover, we have

$$
\left(\tau_{g} \boldsymbol{t}-\kappa_{n} \boldsymbol{b}\right)(s)=\left(0,0, \frac{2 f^{\prime}(s)\left(s^{2}+1\right)+1}{\left(s^{2}+1\right)^{3 / 2}}\right) .
$$

If $2 f^{\prime}(s)\left(s^{2}+1\right)+1 \neq 0$, then $\overline{\boldsymbol{D}}_{o}^{S}(s)=(0,0,1)$ and $\overline{\boldsymbol{D}}_{o}^{L}(s)=\left(-\sqrt{s^{2}+1},-s, 1\right)$. We remark that $\boldsymbol{X}(x, y)=\left(\sqrt{x^{2}+1}, x, 0\right)+y(0,0,1)$ is a cylinder with the director $(0,0,1)$. A cylinder is one of the developable surfaces, so that we now consider general spacelike developable surfaces in $\mathbb{R}_{1}^{3}$. A developable surface $M$ is a ruled surface which is parameterized by $F_{(\boldsymbol{c}, \boldsymbol{\xi})}(t, u)=\boldsymbol{c}(t)+u \boldsymbol{\xi}(t)$, where $\boldsymbol{c}(t)$ is a smooth curve called the base curve and $\boldsymbol{\xi}(t)$ is a smooth curve with $\|\boldsymbol{\xi}(t)\|=1$ which is called the director curve. By definition we have

$$
\frac{\partial F_{(\boldsymbol{c}, \boldsymbol{\xi})}}{\partial t}(t, u)=\dot{\boldsymbol{c}}(t)+u \dot{\boldsymbol{\xi}}(t), \frac{\partial F_{(\boldsymbol{c}, \boldsymbol{\xi})}}{\partial u}(t, u)=\boldsymbol{\xi}(t),
$$

so that the unit pseudo-normal vector at a regular point $(t, u)$ is

$$
\boldsymbol{n}(t, u)=\frac{1}{l}((\dot{\boldsymbol{c}}+u \dot{\boldsymbol{\xi}}) \wedge \boldsymbol{\xi})(t, u)=\frac{1}{l}((\dot{\boldsymbol{c}} \wedge \boldsymbol{\xi})+u(\dot{\boldsymbol{\xi}} \wedge \boldsymbol{\xi}))(t, u),
$$


where $l(t, u)=\left\|\partial F_{(\boldsymbol{c}, \boldsymbol{\xi})} / \partial t \wedge \partial F_{(\boldsymbol{c}, \boldsymbol{\xi})} / \partial u\right\|(t, u)$. We say that $F_{(\boldsymbol{c}, \boldsymbol{\xi})}$ is a developable surface if $\boldsymbol{n}(t, u)$ is orthogonal to $\dot{\boldsymbol{c}}(t)$ for any $(t, u)$. Therefore, the above condition is equivalent to $\operatorname{det}(\boldsymbol{c}(t), \boldsymbol{\xi}(t), \dot{\boldsymbol{\xi}}(t))=0$. Moreover, $F_{(\boldsymbol{c}, \boldsymbol{\xi})}$ is defined to be a spacelike developable surface if $\boldsymbol{n}(t, u)$ is timelike. We remark that $\boldsymbol{\xi}(t)$ is a spacelike vector for a spacelike developable surface. We now consider a curve on $M$ parametrized by

$$
\gamma(s)=\boldsymbol{c}(t(s))+u(s) \boldsymbol{\xi}(t(s))
$$

where $s$ is the arc-length parameter of $\gamma$. Then the unit normal vector along $\gamma$ is

$$
\boldsymbol{n}_{\boldsymbol{\gamma}}=\frac{1}{l}((\dot{\boldsymbol{c}}+u \dot{\boldsymbol{\xi}}) \wedge \boldsymbol{\xi})=\frac{1}{l}((\dot{\boldsymbol{c}} \wedge \boldsymbol{\xi})+u(\dot{\boldsymbol{\xi}} \wedge \boldsymbol{\xi}))
$$

where $l(s)=\left\|\partial F_{(\boldsymbol{c}, \boldsymbol{\xi})} / \partial t \wedge \partial F_{(\boldsymbol{c}, \boldsymbol{\xi})} / \partial u\right\|(t(s), u(s))$. We also have

$$
\begin{aligned}
\boldsymbol{t} & =u^{\prime} \boldsymbol{\xi}+t^{\prime}(\dot{\boldsymbol{c}}+u \dot{\boldsymbol{\xi}}) \\
\boldsymbol{b} & =\frac{1}{l}(\{(\dot{\boldsymbol{c}}+u \dot{\boldsymbol{\xi}}) \wedge \boldsymbol{\xi}\} \wedge \boldsymbol{t}) \\
& =\frac{1}{l}(\langle\dot{\boldsymbol{c}}+u \dot{\boldsymbol{\xi}}, \boldsymbol{t}\rangle \boldsymbol{\xi}-\langle\boldsymbol{\xi}, \boldsymbol{t}\rangle(\dot{\boldsymbol{c}}+u \dot{\boldsymbol{\xi}})) .
\end{aligned}
$$

Moreover, we have

$$
\boldsymbol{n}_{\gamma}^{\prime}=\frac{t^{\prime}}{l}(\ddot{\boldsymbol{c}} \times \boldsymbol{\xi}+\dot{\boldsymbol{c}} \wedge \dot{\boldsymbol{\xi}})+\left(\frac{1}{l}\right)^{\prime} \dot{\boldsymbol{c}} \wedge \boldsymbol{\xi}+\frac{t^{\prime} u}{l} \ddot{\boldsymbol{\xi}} \wedge \boldsymbol{\xi}+\left(\frac{u}{l}\right)^{\prime} \dot{\boldsymbol{\xi}} \wedge \boldsymbol{\xi} .
$$

Therefore, we have

$$
\kappa_{n}(s)=-\frac{t^{\prime 2}(s) d(s)}{l(s)}, \tau_{g}(s)=\frac{t^{\prime}(s) d(s)}{l^{2}(s)}\langle\boldsymbol{\xi}(t(s)), \boldsymbol{t}(s)\rangle
$$

where

$$
d(s)=\operatorname{det}(\dot{\boldsymbol{c}}(t(s))+u(s) \dot{\boldsymbol{\xi}}(t(s)), \ddot{\boldsymbol{c}}(t(s))+u(s) \ddot{\boldsymbol{\xi}}(t(s)), \boldsymbol{\xi}(t(s))) .
$$

Since $\left(\kappa_{n}(s), \tau_{g}(s)\right) \neq(0,0), d(s) \neq 0$ and $t^{\prime}(s) \neq 0$. It follows that

$$
\begin{aligned}
\tau_{g} \boldsymbol{t}-\kappa_{n} \boldsymbol{b} & =\frac{t^{\prime} d}{l^{2}}\left(\langle\boldsymbol{\xi}, \boldsymbol{t}\rangle\left(u^{\prime} \boldsymbol{\xi}+t^{\prime}(\dot{\boldsymbol{c}}+u \dot{\boldsymbol{\xi}})\right)+t^{\prime}(\langle\dot{\boldsymbol{c}}+u \dot{\boldsymbol{\xi}}, \boldsymbol{t}\rangle \boldsymbol{\xi}-\langle\boldsymbol{\xi}, \boldsymbol{t}\rangle(\dot{\boldsymbol{c}}+u \dot{\boldsymbol{\xi}}))\right) \\
& =\frac{t^{\prime} d}{l^{2}}\left\langle u^{\prime} \boldsymbol{\xi}+t^{\prime}(\dot{\boldsymbol{c}}+u \dot{\boldsymbol{\xi}}), \boldsymbol{t}\right\rangle \boldsymbol{\xi} \\
& =\frac{t^{\prime} d}{l^{2}}\langle\boldsymbol{t}, \boldsymbol{t}\rangle \boldsymbol{\xi}=\frac{t^{\prime} d}{l^{2}} \boldsymbol{\xi}
\end{aligned}
$$

so that $\left(\tau_{g} \boldsymbol{t}-\kappa_{n} \boldsymbol{b}\right)(s)$ is parallel to the director curve $\boldsymbol{\xi}(t(s))$.

Proposition 8.1 Let $M$ be a spacelike developable surface parametrized by $F_{(\boldsymbol{c}, \boldsymbol{\xi})}(t, u)=\boldsymbol{c}(t)+$ $u \boldsymbol{\xi}(t)$. For a curve $\boldsymbol{\gamma}(s)=\boldsymbol{c}(t(s))+u(s) \boldsymbol{\xi}(t(s))$ on $M$, the pseudo-spherical osculating spacelike Darboux image along $\boldsymbol{\gamma}$ is $\bar{D}_{o}^{S}(s)= \pm \boldsymbol{\xi}(t(s))$. Moreover, the pseudo-spherical osculating lightlike Darboux image along $\gamma$ is

$$
\bar{D}_{o}^{L}(s)=\frac{1}{l(s)}( \pm \boldsymbol{\xi}(t(s))+\dot{\boldsymbol{c}}(t(s)) \wedge \boldsymbol{\xi}(t(s))+u(s)(\dot{\boldsymbol{\xi}}(t(s)) \wedge \boldsymbol{\xi}(t(s)))) .
$$




\subsection{Curves on the graph of a function}

In this subsection we consider examples similar to those given in [10]. We consider a surface parametrized by $\boldsymbol{X}(x, y)=(f(x, y), x, y)$ with $f(0,0)=0$ and $\partial f / \partial x(0,0)=\partial f / \partial y(0,0)=0$. Here we denote $f_{x}=\partial f / \partial x, f_{y}=\partial f / \partial y, \boldsymbol{X}_{x}=\partial \boldsymbol{X} / \partial x=\left(f_{x}, 1,0\right)$ and $\boldsymbol{X}_{y}=\partial \boldsymbol{X} / \partial y=$ $\left(f_{y}, 0,1\right)$. Since $\boldsymbol{X}$ is a spacelike embedding, we have $\left\|\boldsymbol{X}_{x}\right\|=-f_{x}^{2}+1>0,\left\|\boldsymbol{X}_{y}\right\|=-f_{y}^{2}+1>0$, and a unit timelike normal vector field $\boldsymbol{n}(p)=\frac{\boldsymbol{X}_{x}(u) \wedge \boldsymbol{X}_{y}(u)}{\left\|\boldsymbol{X}_{x}(u) \wedge \boldsymbol{X}_{y}(u)\right\|}=-\frac{1}{\sqrt{1-f_{x}^{2}-f_{y}^{2}}}\left(1, f_{x}, f_{y}\right)$ with $-1+f_{x}^{2}+f_{y}^{2}<0$ where $p=\boldsymbol{X}(u)=\boldsymbol{X}(x, y)$.

We now consider the curve $\gamma(x)=\boldsymbol{X}(x, 0)=(f(x, 0), x, 0)$, where $f(x, y)$ is a smooth function. Here we denote $\frac{d \gamma}{d x}=\dot{\gamma}, f_{x}=f_{x}(x, 0)$, and $f_{y}(x, 0)$. Since $\dot{\gamma}(x)=\left(f_{x}, 1,0\right)$, we have the unit tangent vector field $\boldsymbol{t}(x)=\frac{1}{\sqrt{1-f_{x}^{2}}}\left(f_{x}, 1,0\right)$, and the two unit normal vector fields $\boldsymbol{n}_{\boldsymbol{\gamma}}(x)=\frac{-1}{\sqrt{1-f_{x}^{2}-f_{y}^{2}}}\left(1, f_{x}, f_{y}\right), \boldsymbol{b}(x)=\frac{1}{\sqrt{1-f_{x}^{2}-f_{y}^{2}} \sqrt{1-f_{x}^{2}}}\left(f_{y}, f_{x} f_{y}, 1-f_{x}^{2}\right)$. By straightforward calculations, we have

$$
\kappa_{g}(x)=\left\langle\frac{d \boldsymbol{t}}{d s}(x), \boldsymbol{b}(x)\right\rangle=\frac{-f_{y} f_{x x}}{\left.\left(1-f_{x}^{2}\right)\right)^{\frac{3}{2}}\left(1-f_{x}^{2}-f_{y}^{2}\right)^{\frac{1}{2}}},
$$

and

$$
\kappa_{n}(x)=\left\langle\frac{d \boldsymbol{t}}{d s}(x), \boldsymbol{n}_{\gamma}(x)\right\rangle=\frac{f_{x x}}{\left(1-f_{x}^{2}\right)\left(1-f_{x}^{2}-f_{y}^{2}\right)^{\frac{1}{2}}},
$$

where $s$ is the arc-length. Moreover, we have

$$
\begin{aligned}
\tau_{g}(x)= & \left\langle\boldsymbol{b}(x), \frac{d \boldsymbol{n}_{\gamma}}{d s}(x)\right\rangle \\
= & \frac{1}{\left(1-f_{x}^{2}\right)\left(1-f_{x}^{2}-f_{y}^{2}\right)^{2}}\left\{f_{y}^{2} f_{y x}-f_{x}^{2} f_{y}^{2} f_{y x}+f_{x} f_{y} f_{x x}\right. \\
& \left.+f_{x x} f_{x} f_{y}^{3}-f_{x}^{3} f_{y} f_{x x}-f_{y x}+2 f_{y x} f_{x}^{2}-f_{y x} f_{x}^{4}\right\}
\end{aligned}
$$

We now consider the special case

$$
f(x, y)=a_{20} x^{2}+a_{11} x y+a_{02} y^{2}+a_{30} x^{3}+a_{21} x^{2} y+a_{12} x y^{2}+a_{03} y^{3} .
$$

Then we have $f_{y x}(0,0)=a_{11}, f_{y x x}(0,0)=2 a_{21}, f_{x x}(0,0)=2 a_{20}, f_{x x x}(0,0)=6 a_{30}$. We can show that

$$
\kappa_{g}(0)=0, \kappa_{g}^{\prime}(0)=-2 a_{11} a_{20}, \kappa_{n}(0)=a_{20}, \kappa_{n}^{\prime}(0)=6 a_{30}, \tau_{g}(0)=-a_{11} \text { and } \tau_{g}^{\prime}(0)=-a_{11} .
$$

Since $\kappa_{g}(0)=0$, we can define $\bar{D}_{r}^{S}$ closed to 0 when $\tau_{g}(0)=-a_{11} \neq 0$. It follows that $\delta_{r}^{S}(0)=$ $-a_{20}$ and $\left(\delta_{o}^{S}\right)^{\prime}(0)=6\left(a_{30}-2 a_{11} a_{20} a_{21}\right)$. Therefore, $\bar{D}_{r}^{S}$ is locally diffeomorphic to the ordinary cusp $C$ at 0 if $a_{20}=0$ and $a_{30} \neq 0$. In this case $\bar{D}_{r}^{T}$ and $\bar{D}_{s}^{L}$ cannot be defined closed to 0 because $\kappa_{g}(0)=0$. We can construct examples for $\bar{D}_{o}^{S}$ and $\bar{D}_{o}^{L}$. However, these are rather complicated, so that we omit these. Of course, if we consider a general curve $\gamma(s)=(f(x(s), y(s)), x(s), y(s))$, there might be many other examples. 


\section{References}

[1] V. I. Arnol'd, S. M. Gusein-Zade and A. N. Varchenko, Singularities of Differentiable Maps vol. I. Birkhäuser (1986)

[2] V. I. Arnol'd, The geometry of spherical curves and the algebra of quaternion. Russ. Math. Surv. 50 (1995), 1-68

[3] J. W. Bruce and P. J. Giblin, "Curves and Singularities" (second edition), Cambridge University press, Cambridge (1992)

[4] S. Izumiya, D-H. Pei and T. Sano, Singularities of hyperbolic Gauss maps. Proc. London Math. Soc. 86 (2003), 485-512

[5] L. Chen and S. Izumiya, A mandala of Legendrian dualities for pseudo-spheres in semi-Euclidean space. Proceedings of the Japan Academy, 85 Ser. A (2009), 49-54

[6] S. Hananoi, N. Ito and S. Izumiya, Spherical Darboux images of curves on surfaces. Beitr Algebra Geom., 56 (2015), 675-685

[7] S. Izumiya, Legendrian dualities and spacelike hypersurfaces in the lightcone. Moscow Mathematical Journal 9 (2009), 325-357

[8] B. O'Neill, Semi-Riemannian Geometry, Academic Press, New York, (1983)

[9] I. R. Porteous, Some remarks on duality in $S^{3}$. In : Geometry and topology of caustics, Banach Center Publ. 50, Polish Acad. Sci. (2004), 217-226

[10] T. Sato, Pseudo-spherical evolutes of curves on a spacelike surface in three dimensional LorentzMinkowski space. Journal of geometry 103(2) (2012), 319-331

Noriaki Ito, Sapporo Science Center, Atsubetsu-ku Atsubetsu-chuo 1-5-2-20, Sapporo 004-0051, Japan e-mail:yamagata2009@hotmail.co.jp

Shyuichi Izumiya, Department of Mathematics, Hokkaido University, Sapporo 060-0810, Japan e-mail:izumiya@math.sci.hokudai.ac.jp 